\title{
ARTICLE
}

Cite this: DOI: 10.1039/xoxxooooox

\section{Predictive Design of Engineered Multifunctional Solid Catalysts}

\author{
Robert Raja, ${ }^{a^{*}}$ Matthew E. Potter ${ }^{a}$ and Stephanie H. Newland ${ }^{a}$
}

Received ooth January 2012, Accepted ooth January 2012

DOI: $10.1039 /$ xoxxooooox

www.rsc.org/
The ability to devise and design multifunctional active sites at the nanoscale, by drawing on the intricate ability of enzymes to evolve single-sites with distinctive catalytic function, has prompted complimentary and concordant developments in the field of catalyst design and in situ operando spectroscopy. Innovations in design-application approach have led to a more fundamental understanding of the nature of the active site and its mechanistic influence at a molecular level, that have enabled robust structure-property correlations to be established, which has facilitated the dextrous manipulation and predictive design of redox and solid-acid sites for industrially-significant, sustainable catalytic transformations.

\section{Introduction}

The design of well-defined active centres that are isolated within framework architectures, so as to create discrete single-sites, have long been admired by synthetic and catalytic chemists. ${ }^{1,2}$ The ability to engineer multifunctional sites within these porous architectures with a view to enhancing their catalytic synergy from a structural, functional and mechanistic perspective has since been rationalised ${ }^{3,4}$ affording adequate scope for improving their activity and tuning their selectivity, in industrially significant oxidation and acidcatalysed processes. As fully illustrated and outlined by us earlier, ${ }^{2}$ a "single site" (catalytically active centre) may consist of one or more atoms. These sites are spatially isolated from one another and the activity and functionality of each "site" can be attributable to a particular catalytic function. The choice and proximity of these multifunctional sites coupled with their propensity to modify and tailor the local-structural environment in their immediate vicinity has since instigated the predictive design of novel heterogeneous solids for targeted catalysis. Solid-state chemists have often coveted the intricate design of multifunctional active sites within metalloenzymes and have been intrigued by the efficacy of the threedimensional protein framework in facilitating highly selective and specific reaction pathways. It is believed that each of these singlesites has a distinct role (structural or functional); yet they do not behave as separate entities; there is a clear interplay and synergy, between the two metals sites, which is critical for the catalytic performance. With advances in characterisation and operando methods, synthetic chemists have utilised the knowledge gained from enzymatic active sites to rationally develop porous inorganic frameworks, such as zeolites, as successful mimics with the added advantage of increased stability making the materials more amenable for industrial processing. Extensive research is now on-going, with the aim to mimic the nature and catalytic function of these multinuclear sites, through the use of similar zeotype species containing discrete metal sites to create novel multifunctional catalysts, which could capitalise on the notion of multimetallic synergy.

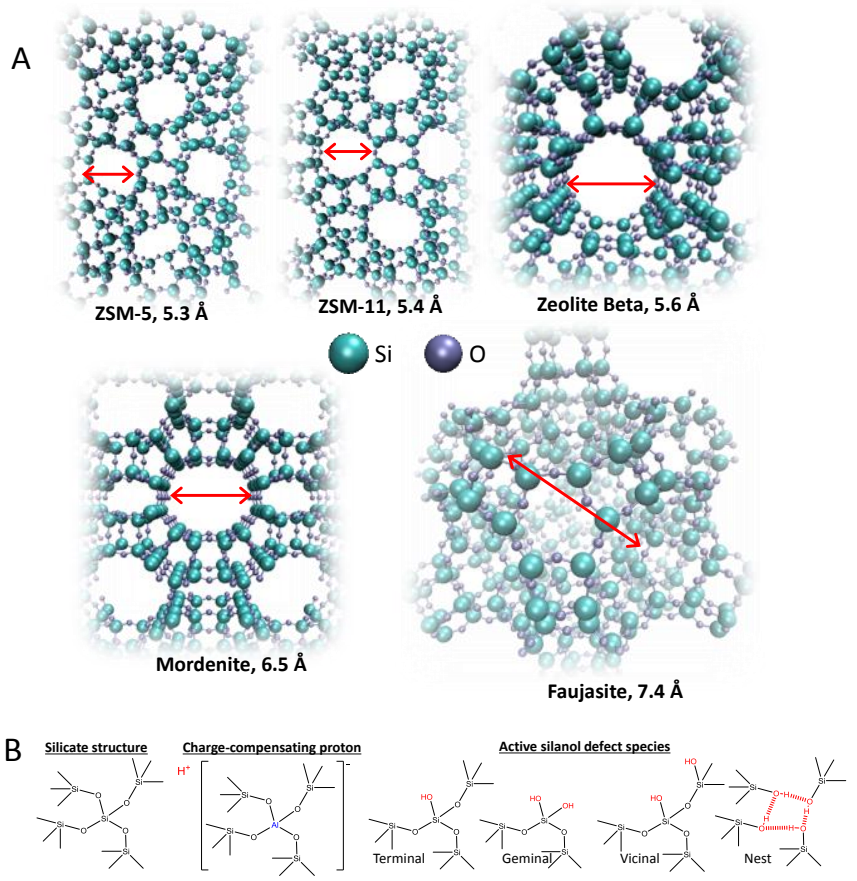

Figure 1: Some industrially-significant zeolite frameworks (A) with designed active centres (B).

A wide-range of over 200 different zeolite frameworks has been successfully synthesised, with numerous other hypothetical architectures yet to be actualised. However, amongst these abundant structures, only a handful have been effectively implemented in industrial processes (Figure 1A) despite the fact that enormous 
progress has been made in novel synthetic approaches coupled with advances in operando spectroscopy.
Scheme 1: Highlighting the origin of charge-balancing cations in zeolites.

Table 1: A selection of solid-acid catalysed processes facilitated by the structures shown in Figure 1.

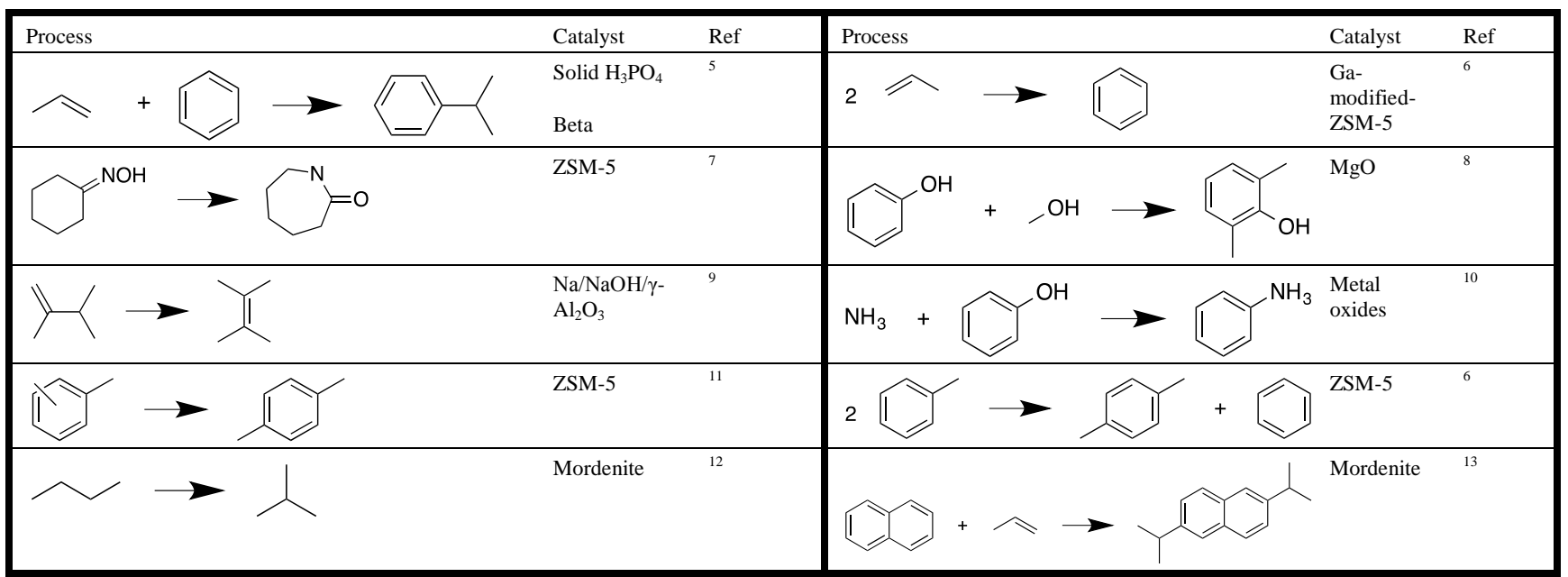

These developments have enabled a diverse-range of active sites to be engineered within these porous architectures, whereby any subtle deviation from the bulk silicate structure can potentially lead to the formation of catalytically active centres. Table 1 shows a selection of acid-catalysed transformations, which have been executed by the types of active centres shown in Figure 1B. These sites have been effective in the industrial implementation of many important acidcatalysed processes in the chemical industry - it is noteworthy that the employment of zeolites is far from sporadic.

Aluminosilicates are most commonly utilised in the petrochemical industry as FCC catalysts and in isomerisation, dehydration and dewaxing applications. The shape-selectivity bestowed on them by the microporous architectures makes them ideal candidates for selectively targeting specific products, whilst simultaneously hindering the formation of bulkier by-products. Furthermore, Table 2 briefly outlines some common examples of industrial oxidation reactions, wherein sacrificial oxidants and peroxides have been employed with solid catalysts. It is noteworthy that this is dominated by 'bulk' metal oxide materials, comprising in general of a larger fraction of less well-defined active centres over atomically engineered, precise single-sites. From a strategic perspective, there is an enormous potential for the predictive design and development of microporous architectures with well-defined, isolated active sites, which are capable of synergistically enhancing both acid- and oxidation-catalysis, with better atom-economy and energyefficiency.

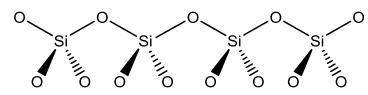

$\mathrm{SiO}_{2}$ species, neutral framework

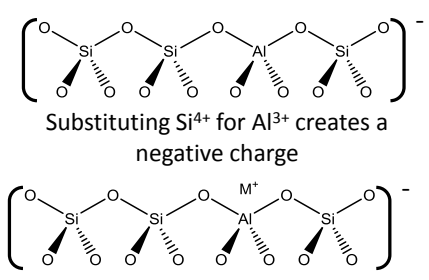

Cations bind to the framework to balance the charge

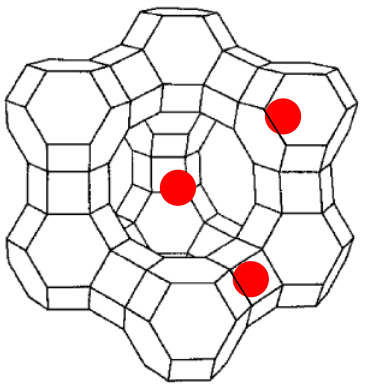

Cations can occupy various positions in the framework.
The mechanism by which active dopant ions are introduced into a zeolitic framework is well understood (Scheme 1). While the incorporation of charge balancing cations is the primary way of generating active centres in zeolitic frameworks, it is also possible to induce catalytic activity using defect sites. While such framework structures are naturally highly crystalline, it is inconceivable that a material could be completely defect free. Figure 1B details a range of defect sites that may commonly occur within the pores and on the surfaces of zeolite catalysts. Note that in all cases such sites arise due to an incomplete second coordination sphere; resulting in a deficient number of $\mathrm{Si}-\mathrm{O}-\mathrm{Si}$ bonds. These sites have been shown ${ }^{7,14}$ to be acidic and efficiently influence acid-catalysed processes. However, the detection of such species can often prove challenging, as the active centres blend with the background bulk structure. Traditionally, such species are detected by indirect spectroscopic methods and most notably via the resultant acidity generated in the material.

It is also possible to generate a much narrower range of active sites through isomorphous substitution, as heteroatoms replace silicon atoms within the porous architecture; although relatively few illustrations of this exist in the literature. ${ }^{15,16}$ Arguably the most well-known example from the past decade that successfully demonstrates the advantages that such a strategy could deliver, both from an academic and industrial perspective, relates to the silicalite family, and specifically to the titanium silicate, TS-1. In this system, a small percentage of the $\mathrm{Si}(\mathrm{IV})$ ions in the silicalite framework (MFI) have been replaced with $\mathrm{Ti}(\mathrm{IV})$ ions. Therefore, instead of $\mathrm{Si}(\mathrm{OSi})_{4}$, a $\mathrm{Ti}(\mathrm{OSi})_{4}$ species exists, acting as an isolated metal-active centre. Given the isoionic nature of the ion substituent, a charge imbalance does not occur from this substitution, and subsequently charge-balancing cations are not required. Instead, the active site is intricately incorporated into the pore walls of the MFI framework and securely anchored within the porous cavity; resulting in a material where the exchange of molecular ions is not readily feasible. This method of creating active sites contrasts the cationic method (Scheme 1) in a number of ways: 
Table 2: Bulk metal-oxides as catalysts in selective oxidation reactions.

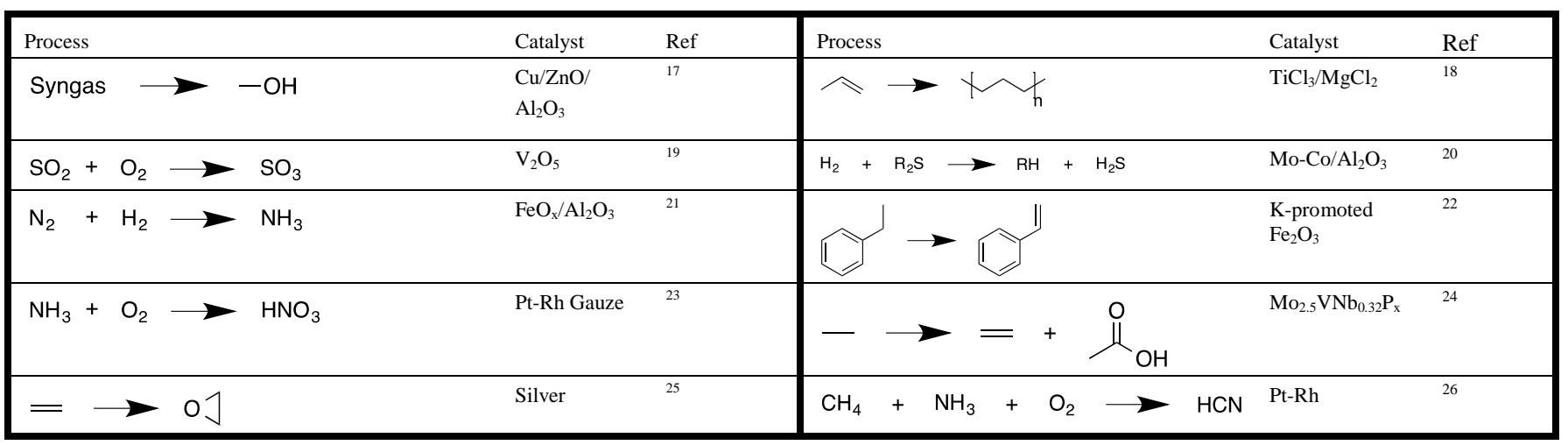

1) The active site can be designed in such a manner that it is truly isolated, thereby making it possible to close the catalytic cycles (loops) and as such automatically regenerates the active centre.

2) When an active site is securely anchored within the framework, it is less susceptible to leaching; thereby facilitating the facile recovery and recyclability of the catalyst.

3) The substituted species can be contorted into an energetically-unfavourable geometry, with the possibility of becoming coordinatively unsaturated and attuning the catalytic activity.

4) Given that additional metals or species are not required for charge-compensation of the overall framework, it minimises the propensity for sidereactions and confers a single-site nature to the catalytically active centre.

Despite these advantages, comparatively few examples of isomorphous substitution in zeolite species exist owing to the stringent restrictions that are imposed on the ions:

a) The substituted species must be of comparable size to the replaced ion, any significant variance would require the framework to become very locally distorted at a great energetic cost.

b) The substituted species must possess an appropriate charge to compensate for the ion it is substituting: a significantly lower charge will result in insufficient electrostatic interactions to keep the ion in the framework and highly positively-charged ions would prompt a net-positive charge on the zeolite, which cannot be accommodated.

c) The substituted species must be able to adopt a tetrahedral geometry within the framework. Isomorphous substitution of a tetrahedral species within the framework will force the substituted species to adopt a similar coordination; though some exceptions are known. ${ }^{3}$

These strict rules within the zeolitic regime led to an expansion in synthetic research to find alternative materials that would be more amenable to framework modification and active site creation. In 1982 a class of microporous materials, aluminophosphates (AlPOs), were discovered, where isomorphous substitution of heteroatoms was readily plausible and is now commonplace. ${ }^{27}$

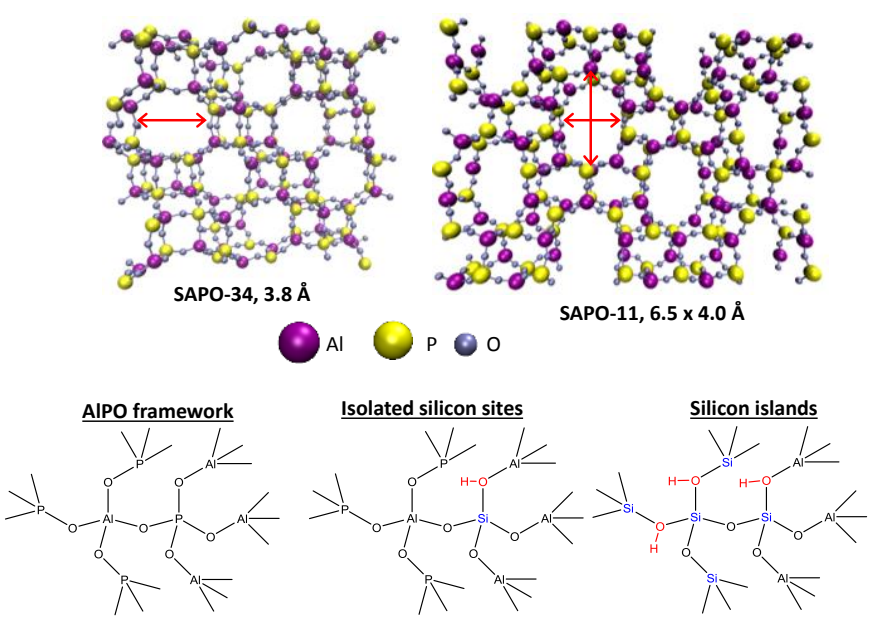

Figure 2: AlPO architectures with silicon-based active-sites.

AlPOs often form analogous frameworks to zeolites, though are not exclusively formed from $\mathrm{Si}$ and $\mathrm{Al} \mathrm{T}$-sites. They are instead derived from alternating $\mathrm{AlO}_{4}$ and $\mathrm{PO}_{4}$ tetrahedra; linking together exclusively through Al-O-P bonds (Figure 2). As a consequence, unlike zeolite materials, the AlPO framework is strictly neutral and is unable to possess any form of net-charge. In light of this, it is not possible to introduce charge-balancing cations and the only method to introduce individual dopant ions into the framework is through isomorphous substitution. The most common example of this is the incorporation of silicon into the AlPO framework (Figure 2). Given the alternating nature of $\mathrm{Al}$ and $\mathrm{P}$ atom tetrahedra within an $\mathrm{AlPO}$, it is logical that one could substitute either an $\mathrm{Al}(\mathrm{III})$ or a $\mathrm{P}(\mathrm{V})$ site, unlike in a zeolite, where only one method of substitution is possible. Subsequently in AlPOs a variety of substitution mechanisms are possible, depending on the oxidation state of the dopant and which framework atom is to be replaced (see Scheme 2). This choice then broadens the scope of possible framework dopants beyond what was possible for a zeolite; as we are now also able to incorporate dopants with $2+$ and $5+$ charges. In spite of the relaxed restrictions on substituent charge, the restrictions on dopant size and coordination geometry still apply. 


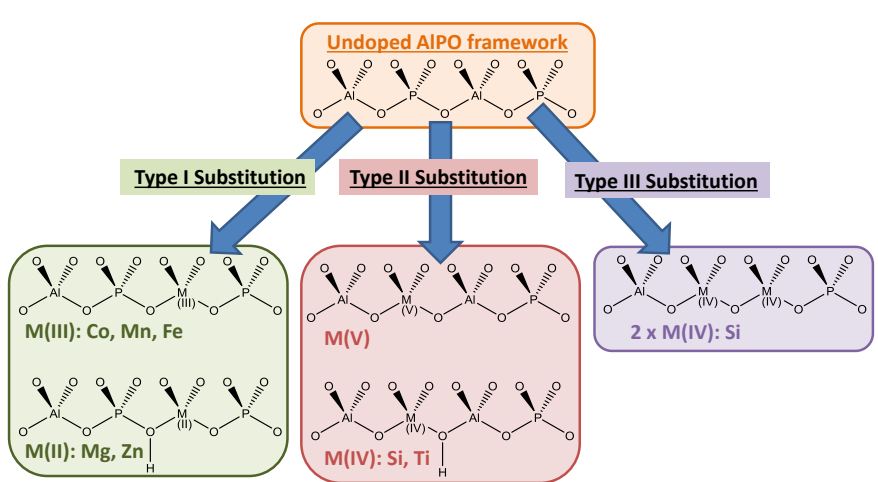

Scheme 2: Typical isomorphous substitution mechanism for the introduction of active sites in AlPOs.

Through adroit choice of the dopant (as with zeolites) it is possible to engineer a range of active sites within the AlPO catalysts. The creation of Brønsted acid sites can be achieved by exploiting the AlPOs inability to hold a net-charge. By undergoing type I substitution with a $\mathrm{M}(\mathrm{II})$ dopant or performing type II substitution with a M(IV) dopant a charge imbalance is created (Scheme 2). However, this imbalance can be neutralised by a proton binding to the framework on an oxygen atom, adjacent to the substituted dopant, thus creating a Brønsted acid site. This synthetic methodology is fundamental in the synthesis of SiAlPO-34, in which the substituted silicon bestows Brønsted acidity onto the framework. The isolated acid sites, in combination with the confining micropores (3.8 $\AA$ ), result in an excellent methanol-to-olefin (MTO) catalyst that has been commercialised for many years. ${ }^{28}$ In a similar manner, one can also engineer redox active sites by selecting metals with two available adjacent oxidation states; commonly these are first-row transition-metals such as $\mathrm{Mn}(\mathrm{II} / \mathrm{III}), \mathrm{Co}(\mathrm{II} / \mathrm{III})$ or $\mathrm{Fe}(\mathrm{II} / \mathrm{III})$. By incorporating these metals into the framework they can then alternate between the two states and operate as an isolated redox centre.

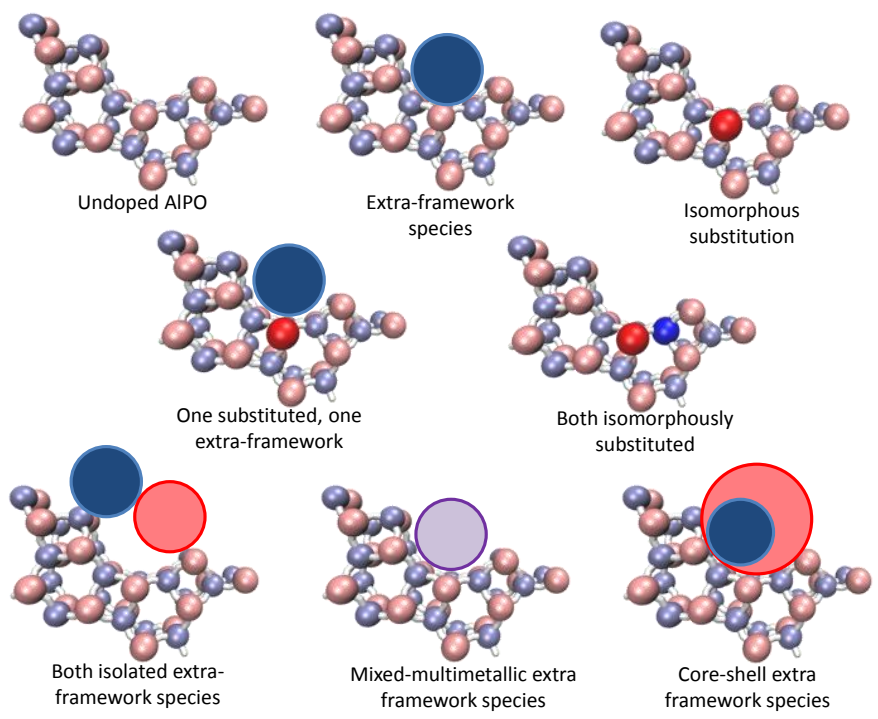

Scheme 3: Potential for multi-metallic substitutions in AlPO architectures.

The limitations on the range of isomorphously substituted sites that can be formed using these synthetic protocols have been previously discussed. ${ }^{29}$ To overcome these restrictions it is possible to extend the range of single-sites present in microporous architectures, by the deliberate application of a range of physical deposition techniques. Therefore, instead of creating isolated active sites, secondary phases are introduced onto and into a host framework, creating species that are more akin to bulk oxides or metallic species. ${ }^{30}$ As isomorphous substitution and physical deposition possess complementary advantages, many literature examples exist ${ }^{31}$ in which the combination of these doping techniques results in improved catalytic performance (see Scheme 3). It has been hypothesised $^{32}$ that the marked catalytic improvements may stem from the deposition process blocking unfavourable substituted sites. Other examples have observed ${ }^{33}$ substituent sites aiding selective impregnation of the bulk oxide and also the stabilisation of specific metal phases.

These microporous molecular sieves have been found to be exceptional catalysts for a range of redox and acid-catalysed processes. Their microporous nature (pore diameters $\leq 20 \AA$ ) only allows specific molecules with appropriate dimensions to access the large internal surface area, this enhances their selective adsorptive capacity, and in turn, their specificity. These catalysts are highly stable $\left(\cong 800{ }^{\circ} \mathrm{C}\right)$ and by adroit choice of aperture-type (cage vs. channel), their selectivity (shape-, regio-, chemo- and enantio-) in demanding catalytic transformations can be suitably tuned. ${ }^{34-36}$ However, due to the interconnecting micropores, diffusion can be hindered for larger organic molecules and this can have a detrimental effect of lower reaction rates and, consequently reduced versatility, for the range of substrates that can access the active sites. Therefore there is a significant interest ${ }^{37}$ into the synthesis of larger-pore zeolites and zeotypes. As a direct consequence, numerous large pore materials have been synthesized, for example, AlPO- $8,{ }^{38}$ VPI- $5^{39}$ and cloverite, ${ }^{40}$ which possess pore diameters in the range of $8-13 \AA$ (see Figure 3 ).

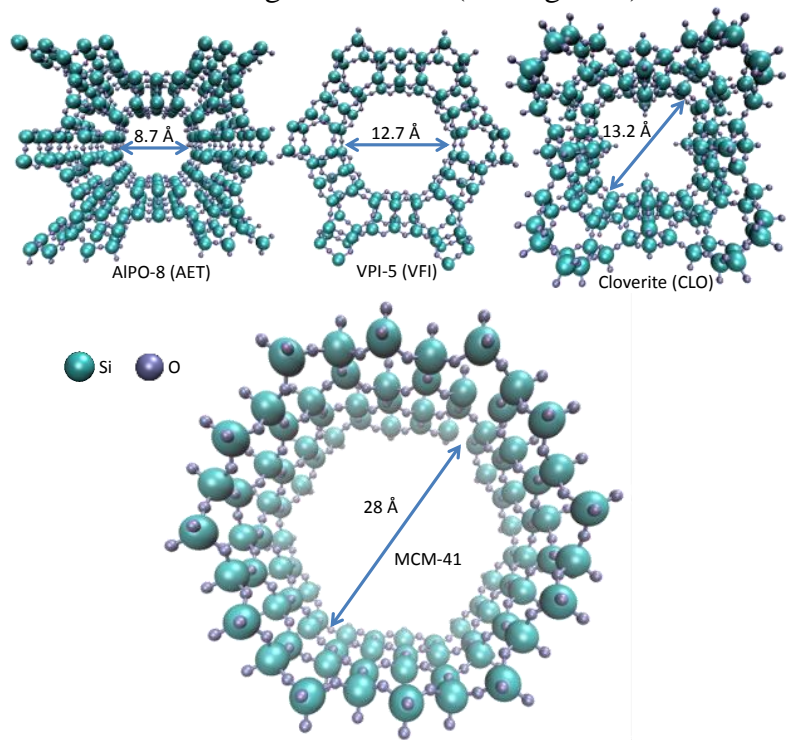

Figure 3: Larger-pore synthetic microporous and mesoporous architectures.

Although it was hoped that the larger pores would result in increased versatility, the resulting materials often consisted of mixed-metal ion coordination sites, terminal $\mathrm{OH}$ groups and resulted in the presence of other non-tetrahedral framework species, which reduced the stability of the materials. ${ }^{41}$ The larger pored molecular sieves were typically amorphous and 
consisted of an irregularly broad distribution of pore sizes, until the first reports for the synthesis of the highly ordered M41S $\mathrm{S}^{42}$ and SBA- $n n^{43}$ and FSM- $n^{44}$ families were presented. MCM-41, SBA-15 and FSM-16 are the most widely cited examples of the mesoporous silicas: they possess a hexagonal array of tuneable uniform mesopores with a narrow pore-size distribution. These mesoporous molecular sieves have attracted considerable attention, due to their increased surface area $\left(\geq 1000 \mathrm{~m}^{2} \mathrm{~g}^{-1}\right)$ without compromising pore uniformity, their long-range ordering and relative stability (compared to other large-pore microporous analogues). The purely siliceous M41S and SBA- $n$ families are comparatively inactive in their undoped form; though once they are doped with an active species, either through isomorphous substitution, deposition, or grafting, they have been shown to yield active catalysts (see Pages 9-13).

The precise environment of the active centre (e.g. oxidation state, coordination number, etc.) at the atomic level is of great significance in the design of active catalysts. The most striking example of an active metal dopant within a microporous zeolitic framework, which has proved effective both from an academic and industrial standpoint, is the four-coordinate titanium active centre in TS-1. The oxophilic Ti(IV) active site in the silicalite framework is the focal point of the well-known TS-1 catalyst that has been commercialised for a range of oxidation applications (Table 3). In an effort to mimic and further optimise the activity of the versatile TS-1 catalyst, a range of titanium-doped silicon materials have been designed and synthesised. Through in-depth characterisation of these systems it has been possible to contrast the exact nature of these different titanium environments, with an aim to understanding the subtleties that underpin the ensuing catalytic activity. With a view to rationalising this, a range of titanium-doped species were investigated using X-ray absorption spectroscopy (XAS), specifically focussing on the X-ray absorption near edge spectroscopy (XANES) region, owing to the low-energy titanium adsorption edge. Through a rigorous study it was revealed that there a significant correlation existed between the XANES pre-edge feature and the coordination number of the titanium site. It was found that ${ }^{45}$ the coordination number could be unequivocally determined by consideration of pre-edge energy value, and the peak intensity through the Farges plot (see Figure 4). Consequently it was found that the reactivity of the TS-1 catalyst can be emphatically associated with the tetrahedral nature of the titanium site. Similarly Ti-MCM-41 (also possessing tetrahedral titanium active centres) has been found to be highly active in a range of oxidation reactions ${ }^{46}$ using peroxides as oxidants. In both cases, the tetrahedral titanium, being coordinatively unsaturated, is able to activate the peroxide species, creating desirable transition-states in the vicinity of the active centre for subsequent activation of the substrate molecule (e.g. an olefin). In stark contrast, five- and six-coordinated titanium species, have not proved to be as effective as their four-coordinate $\mathrm{Ti}(\mathrm{IV})$ analogues ${ }^{47}$ for the reasons outlined below. Five-coordinate titanium active sites are relatively uncommon (given the propensity for titanium to adopt 4- or 6-coordinated geometries), hence the discovery of JDF-L $1^{48}$ was met with great excitement by the heterogeneous catalysis community. Despite this, there are no reports to date of such five-coordinate $\mathrm{Ti}$ species being catalytically superior to the tetrahedral $\mathrm{Ti}$ analogues (TS-1 and TiMCM-41), as it is highly likely that the pyramidal structure ${ }^{48}$ of the active site in JDF-L1 hinders peroxide activation. Similarly octahedral and other 6-coordinate titanium species (such as ETS-4 and ETS-
10) are coordinatively saturated, and as such will retard the interaction of oxidants or reagents, rendering the system comparatively inert. ${ }^{49}$ This link between coordination geometry and catalytic activity further emphasises the notion that a profound knowledge of the precise nature of the active site at the molecular level is of fundamental importance in the design of highly active catalysts, and synthetic factors that provoke even a subtle modification to the nature of the active site at the atomic scale deserves a thorough investigation.

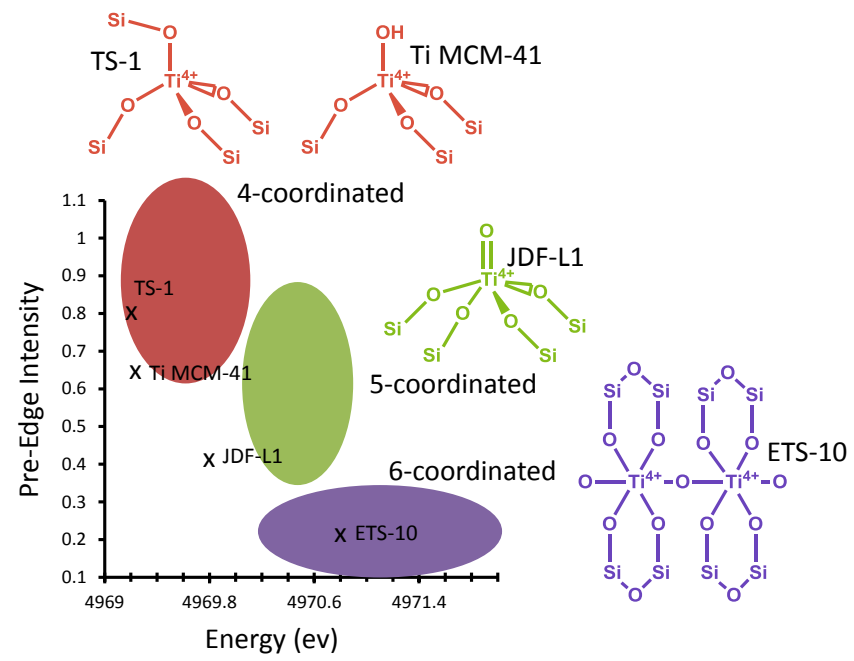

Figure 4: Using XANES for probing the local structural geometry and coordination environment in solid catalysts. ${ }^{45}$

The need to adeptly design specific active sites is already wellestablished within nanoparticle chemistry; where intrinsic compositional control at the nanoscale coupled with tuning the size- and shape- of the metal nanoparticles, ensues the origin of discrete single-sites that are catalytically active and selective (see Table 3)..$^{50-52}$ A wide-range of synthetic strategies ${ }^{53}$ have been recently evolved to generate metal nanoparticles with innate control on morphology and particle size. One such method involves the introduction of carbonyl-capped metalnanoclusters to solid (typically siliceous) supports to form particles of specific sizes and molecularity. ${ }^{54}$ A similar synthetic strategy can be used to form well defined, isolated active sites and has been demonstrated by grafting titanocene dichloride onto the inner walls of M41S, to generate the Ti MCM-41 catalyst. $^{46}$ Here, instead of using a complex nanocluster as a precursor, an isolated organometallic species (titanocene dichloride) was used. Whilst such a strategy has been highly effective at the laboratory scale, industrial scale-up for bulk chemistry applications has proved expensive and often difficult to manipulate, due to the delicate air-sensitive nature of the reagents and precursors. Recently in situ methods of generating metal nanoparticles have been developed ${ }^{55}$ through the addition of an anionic metal-chloride precursor into the pores of a copper chloropyrophosphate framework. Using this approach, precursors of $\mathrm{Pt}, \mathrm{Pd}$ or $\mathrm{Au}$ were extruded from the pores of the support, to generate isolated metal nanoparticles for the aerobic oxidation of benzyl alcohol (Table 3). 
Table 3: The origin of single-site solid catalysts for effecting highly demanding chemical transformations with high selectivity. $\mathrm{C}=$ Conversion, $\mathrm{S}=$ Selectivity.

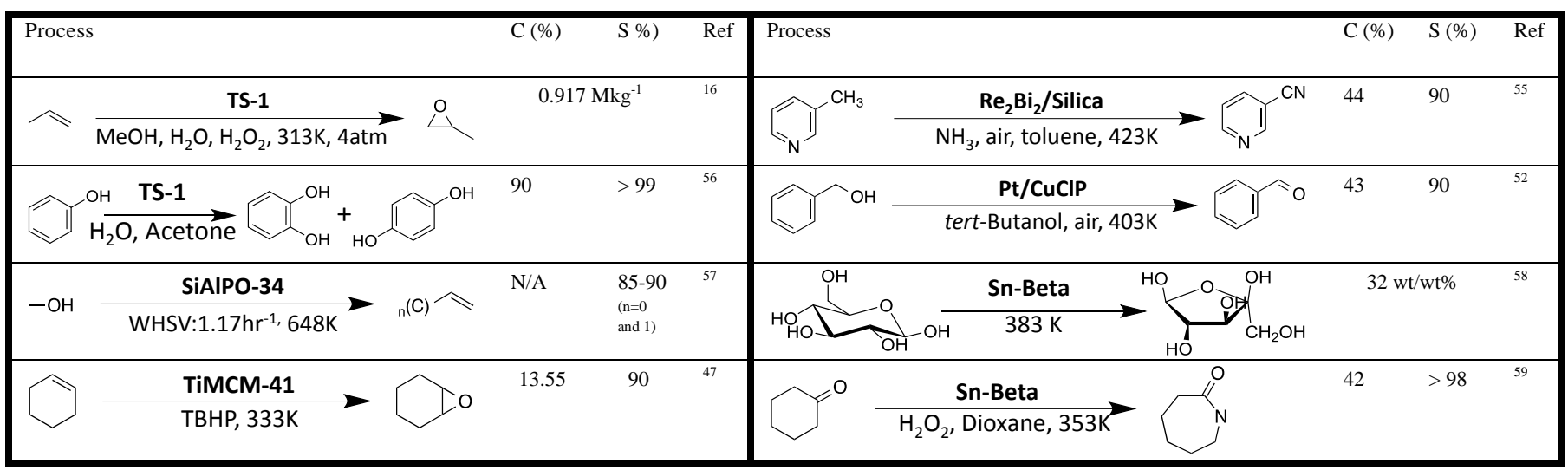

Despite the range of post-synthesis techniques that have been well established and developed ${ }^{60}$ by the zeolite community to tune framework properties, (such as de-alumination and tethering silanol species to tune the hydrophobicity, to highlight a few) such techniques are not easily translated to their AlPO (or zeotype) counterparts. As such, alternative synthetic strategies must be developed to allow for subtle modifications (oxidation state, coordination geometry, substitution mechanism) of the active centre in the AlPO framework. In this vein, there has been immense interest over the last decade in combining different metal dopants to modify the catalytic profile; thus generating multifunctionality and synergistic enhancements within AlPO architectures. More specifically active sites (Scheme 2) can be engineered to bestow targeted catalytic properties in the AlPO framework, through careful choice of dopant precursors, with a view to predictively designing and enhancing the catalytic potential to confer redox and/or acidic capabilities. Though this approach, one is not limited to choosing just one active site, and can in-fact construct a range of different catalytic centres in one system; thereby creating a multifunctional catalytic system. An example of this details the use of a bimetallic AlPO catalyst for the formation of the Nylon-6 precursor, $\varepsilon$-caprolactam, in a singlestep from cyclohexanone (Figure 5A). ${ }^{61,62}$ In the initial step, ammonia and air are converted into hydroxylamine (in situ) inside the pores of the AlPO catalyst, a step requiring a redoxactive site. The hydroxylamine formed, consequently converts the cyclohexanone to cyclohexanone oxime. ${ }^{63,64}$ In the secondstep of this process, the oxime undergoes a Beckmann rearrangement to yield the desired $\varepsilon$-caprolactam, utilising the Brønsted-acid from the same bimetallic catalyst. ${ }^{65}$ Therefore, in order to successfully tailor a catalyst for this demanding onepot process the catalyst requires two different active sites. This was achieved ${ }^{64}$ by introducing manganese and magnesium into the same framework to create a bimetallic $\mathrm{Mn}$ (III)Mg(II)AlPO5 catalyst, which was shown to produce $\varepsilon$-caprolactam in a sustainable fashion. The two active centres in the AlPO framework can be thought of as independent single-sites, with each site performing a separate targeted function; $\mathrm{Mn}$ (III) ions executing the ammoximation step and $\mathrm{Mg}$ (II) ions enabling the Beckmann rearrangement. Given that both active centres undergo type I substitution (see Scheme 2), there is very little possibility that the two sites will be in close proximity to one another, and as such, will have little influence on each other; hence they can be considered "truly isolated".
The benefits of bimetallic substitution which result in the above bifunctionality, which while elegant for cascade processes in AlPOs, can be extended further to optimise individual reaction steps, with a view to enhancing the synergistic aspects associated with the catalysis. The introduction of a second metal can also regulate the properties of the individual active sites, when the two metal centres are able to interact favourably with each other (cooperative effect). An example of this is depicted in the epoxidation of cyclohexene (Figure 5B), with a bimetallic $\mathrm{Co}$ (III)Ti(IV)AlPO-5 catalyst. In this case, the bimetallic Co(III)Ti(IV)AlPO-5 catalyst showed a significant improvement in catalytic potential over both of its corresponding monometallic analogues, Co(III)AlPO-5 and Ti(IV)AlPO-5. This intriguing phenomenon was probed further by means of additional experiments to verify the concerted aspects associated with this catalysis and contrast it with synergistic enhancements. It was hypothesised ${ }^{4}$ that this improvement in catalytic potential may not be genuine synergy and could instead simply be the result of a concerted combination of cobalt and titanium being present in the same reaction mixture. To explore this theory, the activity of a physical mixture of Co(III)AlPO-5 and Ti(IV)AlPO-5 was also investigated, under identical conditions, utilising analogous metal concentrations as in the bimetallic analogue. The results ${ }^{4}$ were fairly conclusive in that the observed synergistic enhancement was genuine, and further, only occurred when both metals were in the same framework (proximity effect). The nature of this observed catalytic synergy was probed using in situ UV/Vis spectroscopy (and more recently using EPR spectroscopy $)^{3}$ which revealed that the titanium site was significantly more tetrahedral in the bimetallic Co(III)Ti(IV)AlPO-5 catalyst, than in its corresponding monometallic Ti(IV)AlPO-5 analogue. ${ }^{66}$ It was therefore concluded that the simultaneous incorporation of $\mathrm{Co}(\mathrm{III})$ and Ti(IV) ions results in a subtle modification of the local structural environment around the titanium centre, which resulted in marked improvements in catalytic activity.

It is important and this stage to reflect on, and contrast the above two examples: In both cases a redox active centre and a Brønsted-acid site have been incorporated into the same AlPO framework that enhanced the yield of the desired product. In the $\varepsilon$-caprolactam example, the $\mathrm{Mn}$ (III) and $\mathrm{Mg}$ (II) sites are, as already stated, operating in completely isolated fashion, each performing a unique role in different reaction steps. In the epoxidation example, however, the $\mathrm{Co}(\mathrm{III})$ and $\mathrm{Ti}(\mathrm{IV})$ sites are both involved in the same reaction step and are influencing 
each other. The simultaneous incorporation of both metals (during synthesis) here creates subtly different sites than those present in the monometallic analogues. These species are found to be more active in enhancing the epoxidation reaction and a synergistic relationship is present between the two active centres at the molecular level, which extends beyond the scope of bifunctionality.

Further examples of synergistic relationships have been reported ${ }^{67}$ with mesoporous silica catalysts. MnTi-SBA-15 was found to have a much higher activity and selectivity in the oxidation of ethyl benzene than its corresponding monometallic analogues and the physical mixture (Figure 6). ${ }^{67}$ The high catalytic activity is attributed to the simultaneous incorporation of Mn(II) and Ti(IV), as confirmed by UV-Vis spectroscopy. It has been further demonstrated that the two species are in close proximity to each other, thereby enabling the two active centres to act in a coordinated fashion. This results in the oxophilic Ti(IV) ions coordinating with the oxidant which is in close proximity to the redox-active site (Mn) that interacts with the substrate. The close proximity of the two sites results in a significant enhancement in catalytic activity, given the EleyRideal nature of the process. ${ }^{68}$ The synergistic phenomenon is comparable to the findings by Paterson et al with the microporous Co(III)Ti(IV)AlPO-5 catalyst. ${ }^{4}$

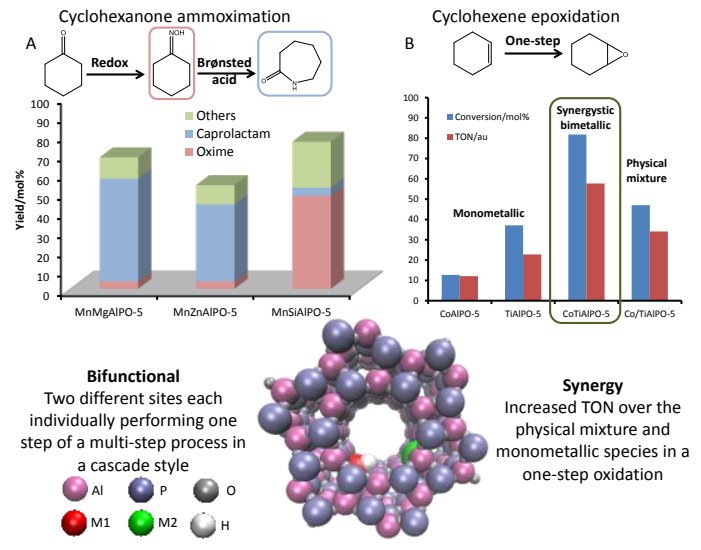

Figure 5: Contrasting multifunctionality (A) with catalytic synergy $(\mathrm{B}) .^{4,64}$

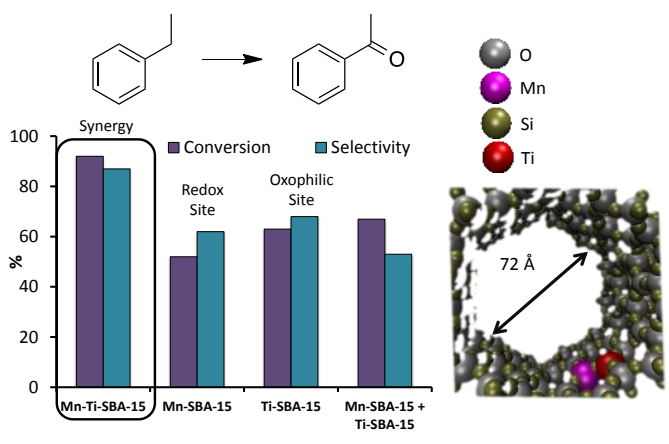

Figure 6: Effecting catalytic synergy in mesoporous architectures for the oxidation of ethyl benzene. ${ }^{67}$

\section{Probing the Nature of the Active Site at the Molecular Level}

Redox-active sites for selective oxidation

In order to gain a comprehensive and holistic understanding of the nature of the active sites and their precise location and geometry within the framework, it is necessary to employ a wide-range of structural and spectroscopic characterisation tools. Robust structure-property relationships are essential in the rational design of single-site catalysts and these can be realised by utilising an array of ex situ, in situ and operando physico-chemical structural techniques. The introduction of a dopant species into the framework, can often distort the unit cell and, in extreme situations, can disrupt the frameworks crystallinity. In some cases, an increase in cell parameters can be observed after isomorphous substitution; which can be rationalised to the successful isomorphous incorporation of the dopant ${ }^{67,69}$ However, in many cases even with the incorporation of a heavy-metal such as ruthenium (see Figure 7C), these effects are notably subtle (given the low-level of metal substitution) and higher-resolution synchrotron radiation is required to observe such an expansion. ${ }^{70}$ Whilst XRD is fundamental for establishing crystallinity and phase-purity, detailed information on the textural characteristics and micro/meso-porosity can be ascertained using the BrunauerEmmett-Teller (BET) method of analysis. ${ }^{71}$ Scanning electron microscopy (SEM) and transmission electron microscopy (TEM) are both valuable for determining morphological features, compositional integrity and uniformity, particle size distributions and evolution of porosity, with recent developments in aberration-corrected microscopy affording quantitative information at the atomic scale. ${ }^{70}$
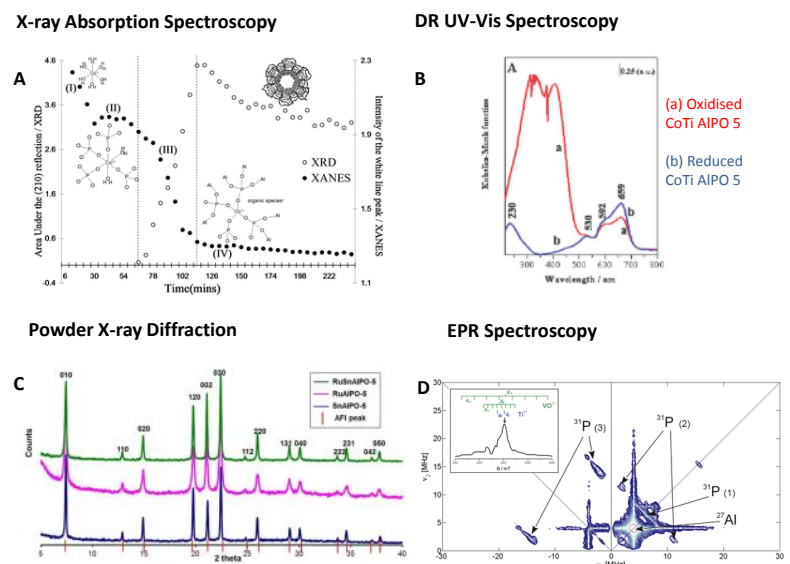

Figure 7: Establishing structure-property relationships through the use of in situ spectroscopic and structural characterisation methods. ${ }^{3,4,70,72}$

In situ diffuse reflectance (DR) UV-Vis spectroscopy is another useful tool for ascertaining the local environment of the metal centres; it is of particular use when examining the redox capabilities of the transition metals and their coordination environments. The presence of certain spectroscopic features can ascertain the extent to which transition metals are incorporated into a framework. For example, the presence of manganese (II) substitution into SBA-15 was confirmed by the band at $270 \mathrm{~nm}$, which corresponds to a ligand to metal charge transfer (LMCT) $\mathrm{O}^{2-} \rightarrow \mathrm{Mn}^{3+}$ in a tetrahedral environment. However, it was also noticed that there were some extraframework Mn(III) species present (band at $513 \mathrm{~nm}$ ) which could be attributed to d-d transition of $\mathrm{Mn}^{2+}$ in octahedral geometry. ${ }^{67}$ This prompted further research into optimising the synthetic strategy, that eventually led to the development of a more active catalyst. ${ }^{67}$ The position of the bands can also indicate the oxidation state (Figure 7B) of the transition-metal: for example tetrahedral Co (III) LMCT transitions occur in the 
250nm-500nm range; whereas the $\mathrm{d}$ - $\mathrm{d}$ transitions that ensue in the tetrahedral, reduced Co (II) species manifest as a triplet at 530, 592 and $659 \mathrm{~nm}$ respectively (see also Page 6). ${ }^{4}$

XAS is also a powerful tool for probing the local structural environment of a selected element, regardless of symmetry or periodicity; and can be employed to determine the crystallisation mechanism, coordination geometry, oxidation state, substitution mechanism and redox reversibility of the metal species. This technique can be used to allude to the exact nature of the metal at the atomic level and this can provide valuable insights into the catalytic behaviour for developing robust structure-property relationships. Simmance et $a l^{72}$ have developed an in situ combined powder-XRD and XAS technique to probe the substitution of cobalt species into an AlPO-5 framework during the crystallisation stage. XRD was utilised to probe the long-range order of the microporous catalyst and it was found that after 66 minutes of the hydrothermal synthesis, the crystalline AlPO-5 phase had formed. A simultaneous comparison of the XRD and Co-K edge XANES features enabled the elucidation of a possible crystallisation mechanism (Figure 7A), in which the transformation of octahedral $\mathrm{Co}$ (II) ions into their tetrahedral coordination prior to crystallisation was clearly observed. ${ }^{72}$

EPR is also a potent technique to probe the local structural environment of paramagnetic transition-metal ions. ${ }^{73}$ In partnership with our collaborators, ${ }^{3,74}$ we have employed continuous wave $(\mathrm{CW})$ and electron spin echo measurements to elucidate the oxidation states and coordination geometries of our CoTiAlPO-5 and VTiAlPO-5 catalysts, that were particularly active in selective oxidation reactions. In order to quantitatively probe the local environment of the individual metal ions, with a view to studying the nature of the transitionmetal substitution mechanism (see Scheme 2), an array of hyperfine techniques (Figure 7D) associated with EPR can be utilised; namely Electron Spin Echo Envelop Modulation (ESEEM), Electron Nuclear Double Resonance (ENDOR), Hyperfine Sublevel Correlation (HYSCORE). HYSCORE allows the interaction of the unpaired electron with the magnetically active nuclei of the AlPO lattice $\left({ }^{31} \mathrm{P}(\mathrm{I}=1 / 2),{ }^{27} \mathrm{Al}\right.$ $(I=5 / 2)$ to be assessed - see Page 11 for further discussions on the structure-property correlations that were established by using this technique in combination with the catalytic results.

\section{Deciphering the nature and strength of solid-acid centres}

Given that zeolites and similar zeotype materials are primarily employed as solid-acid catalysts in the chemical industry, there has been great deal of interest in developing protocols and methods for exploring these specific active sites. Fouriertransform infra-red spectroscopy (FT-IR) is routinely used to qualitatively correlate the bands associated with the $\mathrm{O}-\mathrm{H}$ stretches to the acid-site strength. However, in order gain detailed quantitative information on the nature and strength of solid acid centres, it becomes imperative to employ a suitable probe molecule (such as $\mathrm{CO}$ ) for quantifying the nature and type of such sites (Figure 8A). It is often beneficial to combine the information garnered from FT-IR with other spectroscopic methods, such as temperature-programmed desorption (TPD), to complement and categorise the respective fractions of 'strong', 'weak' or 'intermediate' acid sites present on a specific species. In our studies ${ }^{71,75}$ that were particularly aimed at establishing structure-property correlations in the industrially significant Beckmann rearrangement of cyclohexanone oxime and in the isopropylation of benzene to cumene, it was important to establish selection rules for the classification and quantification of the Brønsted acid centres present in our catalysts.

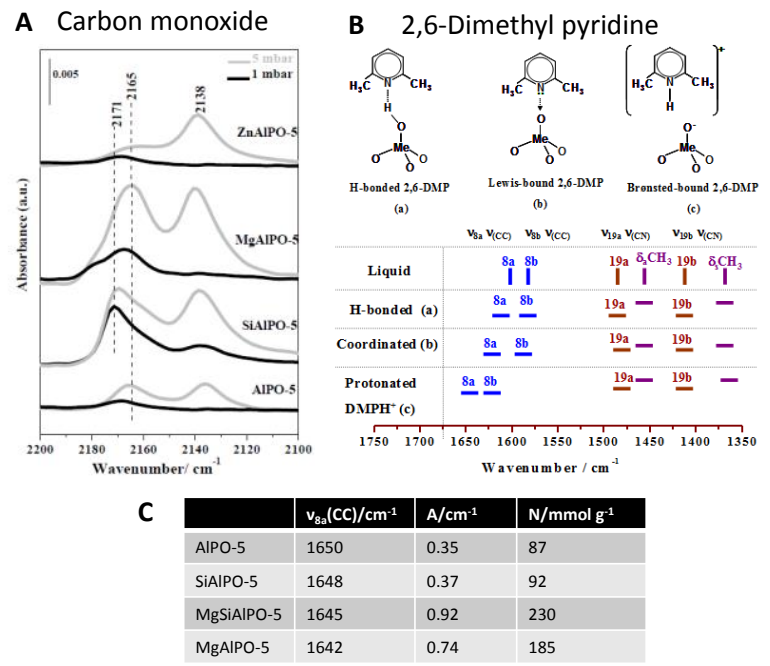

Figure 8: Detailing the use of molecular probes (CO and 2,6DMP) for quantifying nature and type of solid-acid centres. ${ }^{71,75}$

The TPD measurements on our solid acid catalysts ${ }^{71}$ were in good agreement with the FTIR findings on the position of the $\mathrm{v}_{8 \mathrm{a}}(\mathrm{CC})$ bands (Figure 8C), but it was difficult to decipher subtle differences between the monometallic and bimetallic solid acid catalysts used in this study ${ }^{71}$ purely on the basis of the $\mathrm{NH}_{3}$-TPD data. It is to be noted that the $\mathrm{NH}_{3}$ probe that was used in the TPD measurements bears little resemblance to the substrates (see Page 9 for catalytic data) used in our study ${ }^{71}$ and it was more pertinent to employ a probe-molecule that bears a greater resemblance to the reagents, so that only the catalytically relevant species may be observed. In light of this, we resolved and quantified the FTIR signals observed using the 2,6-Dimethylpyridine (2,6-DMP) as a molecular probe (see Figure 8B), and this not only highlighted the subtle differences in acid-site strength that existed between our catalysts; but more importantly emphasised the differences in the quantity of accessible active (acid) sites. We believe that the quantification of the number and strength of acid sites using 2,6-DMP as a probe is invaluable in determining the difference in acidic density, with the TPD providing complementary evidence for these species. By gaining a thorough understanding of the precise nature and strength of the solid-acid active centres, it has been possible to predict the type of substitution mechanism (Scheme 2) that leads to the creation of isolated single-sites for targeted catalysis.

While the above spectroscopic techniques produce a wealth of information about the nature and strength of the acid-species present, they reveal very little information on the local environment around the active site. This is something that is of fundamental importance and must be well understood for the rational and targeted synthesis of active sites in the future. Preliminary efforts ${ }^{65}$ using 1D and 2D MAS ${ }^{29} \mathrm{Si}$ NMR have provided valuable quantitative insights into the Si-substitution mechanism (type II versus type III - see Scheme 2) within these molecular sieves, which also provide direct evidence for 
the formation of isolated $\mathrm{Si}$ sites and silicon islands that are responsible for regulating the catalytic activity and selectivity. While modern characterisation tools and operando spectroscopy can provide detailed information on the framework and the types of active sites; there are some limitations on the degree of information they can provide on an electronic scale. This is particularly relevant when considering dynamic systems. Recent developments in computational chemistry have provided some of the necessary tools that are required to gain quantitative insights into the nature of a diverse range of catalytically active single sites. ${ }^{76}$ There are many complimentary theoretical techniques such as cluster-based and periodic DFT, ${ }^{76}$ Monte-Carlo simulations, molecular dynamics and QM/MM models that are currently employed in solid-state chemistry (particularly relating to porous solids), ${ }^{77}$ which when allied with sophisticated operando characterisation afford a valuable tool for affording structure-activity relationships.

\section{Predictive Design and Structure-Property Relationships in Oxidation and Acid Catalysis}

\section{Solid-Acid Catalysis}

Un-doped AlPOs and a vast proportion of mesoporous silicas are charge neutral and hence are not naturally acidic; hindering their direct application in acid-catalysed transformations. Judicious incorporation of transition-metal ions into the inner walls of the nanoporous framework can result in careful tailoring of the acidity by meticulous consideration of the valency and the loading of a chosen metal ion. Acidity, in some cases, can be enhanced by increasing the concentration of the active centres or type of substitution mechanism (see Scheme 2) and can lead to an increase in catalytic performance. ${ }^{78}$ Although, sufficient caution should be exercised when introducing transition-metals into the framework; if the loading is too high it can promote the transformation of metal ions into extra-framework metal oxide species, resulting in undesired side-reactions. $^{79}$

Two examples of bi-substitution enhancement in catalytic efficiency and synergy are depicted in Figure 9A \& B, which employ contrasting incorporation methods and type of substitution (from Scheme 3). The first example refers to the introduction of zinc into a catalytically active monometallic MgAlPO-5 catalyst to create a bi-substituted entity. It was found that the monometallic ZnAlPO-5 catalyst was catalytically inactive for both the isopropylation of benzene and the vapour-phase Beckmann rearrangement of cyclohexanone oxime. These two reactions require different (contrasting) types of acid centres: the former is known to require strong acid sites, whereas the latter is known to favour weak acid sites. Therefore, inactivity for both reactions suggested a widespread absence of Brønsted acid centres in the ZnAlPO-5 catalyst. This was confirmed using in situ FTIR spectroscopy employing CO as a molecular probe, which compared the findings ${ }^{71,75}$ from undoped AlPO-5 through to the mono-substituted and bisubstituted analogues shown in Figure 9. This technique showed the only acidity present in the monometallic $\mathrm{ZnAlPO}-5$ was derived from the ubiquitous $\mathrm{P}-\mathrm{OH}$ defect sites, which were present in near-identical quantities in this and the up-doped system. This confirmed that no acid sites had been generated on addition of the zinc dopant by the hydrothermal synthesis procedure. Powder-XRD suggested that instead of under-going isomorphous substitution, zinc had formed extra-framework $\mathrm{ZnO}$ species, which further elucidates the lack of activity. On the other hand, using $\mathrm{Mg}$ as the dopant, the resulting monometallic MgAlPO-5 (known to produce strong acid sites) showed good activity in the isopropylation reaction, and likewise the monometallic SiAlPO-5 was effective for the Beckmann rearrangement of cyclohexanone oxime. By extending the in situ CO-probed FT-IR study, it was found that the total number of acid sites had increased on doping the framework with these metals ( $\mathrm{Si}$ and $\mathrm{Mg}$ ), further confirming their isomorphous incorporation into the framework. In addition to using $\mathrm{CO}$ as a molecular probe, the acid strength was also specifically investigated using a 2,6 dimethyl pyridine (2,6DMP) as the molecular probe (Figure 8 ); which unequivocally showed the presence of a higher proportion of stronger Brønsted acid sites in the MgAlPO-5 and comparatively weaker ones in the SiAlPO-5 catalyst, in good agreement with the catalysis results (Figure 9A \& B) and consistent with previous published work. ${ }^{80}$

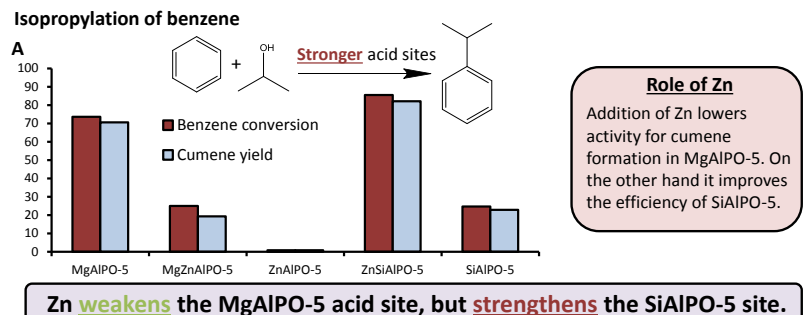

Zn weakens the MgAIPO-5 acid site, but strengthens the SiAIPO-5 site.

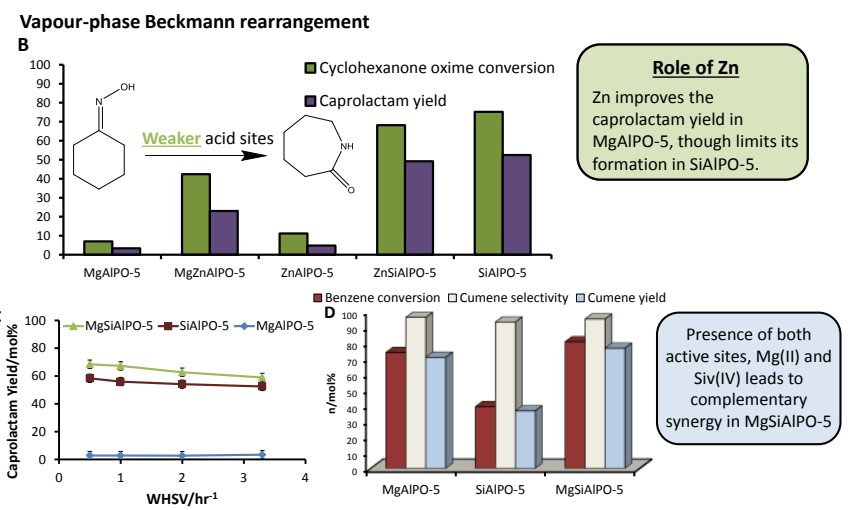

Figure 9: The role of $\mathrm{Zn}$ in modifying the acid strength (A, B); and facilitating catalytic synergy in bimetallic $\mathrm{Mg}(\mathrm{II}) \mathrm{Si}$ (IV)AlPO-5 for acid catalysis (C, D). ${ }^{71,75}$

Despite the inactivity of zinc in the framework, a bimetallic MgZnAlPO-5 and ZnSiAlPO-5 species were synthesised. This required the development of a one-step hydrothermal synthesis protocol to influence and optimise the catalytic profiles of both reactions and to further contrast the findings with the monometallic catalysts. The addition of zinc to the magnesiumdoped catalyst resulted in an unsurprising decrease in activity for the isopropylation of benzene (Figure 9A). Given the inert nature of the zinc in the monometallic species, this is consistent with our observations. However, in stark contrast, the yield of $\varepsilon$-caprolactam was significantly improved with the bimetallic MgZnAlPO-5 catalyst over that of its corresponding monometallic analogues (Figure 9B). These observations were transposed for the ZnSiAlPO-5 system: the bimetallic ZnSiAlPO-5 showed significant improvements in the isopropylation of cumene, though the introduction of $\mathrm{Zn}$ had a detrimental effect in the acid-catalysed Beckmann rearrangement. 
Table 4: Enhancing catalytic synergy in acid-catalysis through the deployment of bimetallic active sites in micro- and meso-porous solids. $\mathrm{C}=$ Conversion, $\mathrm{S}=$ Selectivity

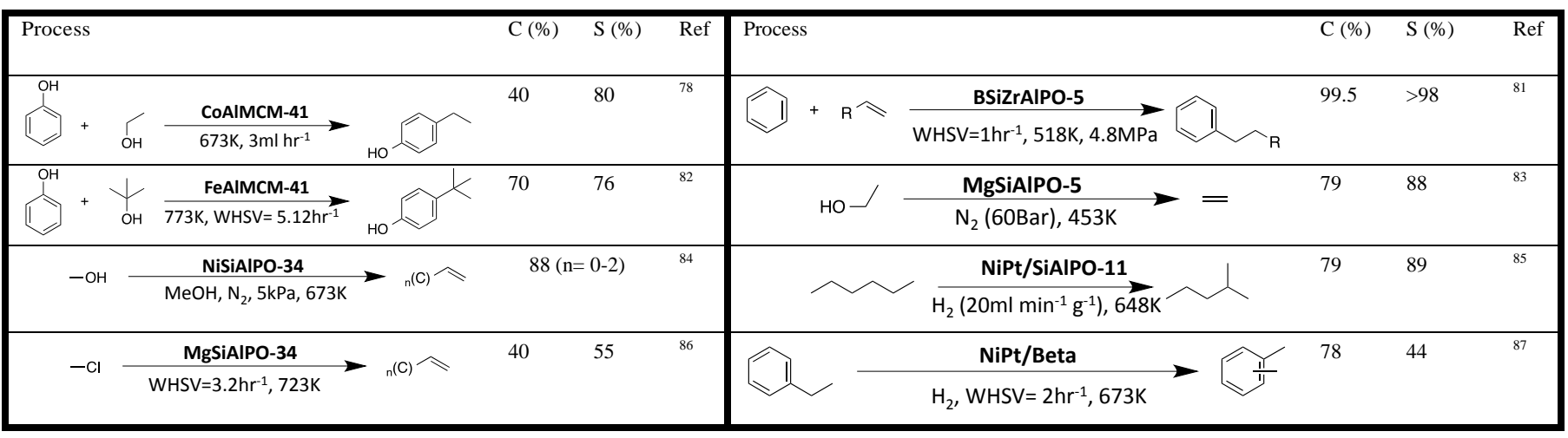

With a view to fully rationalising these findings, a comprehensive spectroscopic study was undertaken ${ }^{75}$ to understand the remarkably contrasting effects of adding zinc to the monometallic catalysts. Again, through the use of the 2,6DMP as a molecular probe, it was found that the addition of $\mathrm{Zn}$ had weakened the acid site strength of the magnesium sites; whilst it had modified and somewhat strengthened the acid strength associated with the silicon sites, in accordance with the catalytic findings. It was concluded from these studies ${ }^{71,75}$ that through the inclusion of zinc in a microporous framework architecture, it becomes possible to subtly modify the (acid) strength of different sites. Furthermore, it was revealed ${ }^{75}$ that zinc is capable of provoking different catalytic responses from different acid sites; this therefore provides a new tool for tuning acidity and targeting catalytic outcomes by predictively designing active centres.

The contrasting catalytic behaviour of the monometallic MgAlPO-5 and SiAlPO-5 catalysts, provoked further interest in combining the two dopants into one framework to form a bimetallic MgSiAlPO-5 entity. As it has been already established $^{80,88}$ that both dopants could generate acid sites with diverse acid strengths through framework substitution, it was rationalised that a combination of these two different active sites was of great interest in optimising the design strategy, whilst simultaneously increasing its versatility. Synthetic approaches were rigorously designed to create a bimetallic MgSiAlPO-5 catalyst, and in doing so it was found that, the presence of two different acid sites within the same framework was beneficial in both the isopropylation and Beckmann rearrangement reactions (Figure 9C \& D). It was noteworthy that the catalytic outcome with the bimetallic analogues surpassed that of the monometallic catalysts in both cases. This synergistic enhancement was attributed to the concerted effect of having two different types of acid sites with intrinsic acid strengths, and each of these individual acid sites contributing to different steps in the multi-step reaction pathway, thereby facilitating improvements in both reactivity and product selectivity. Other related examples involving bimetallic and trimetallic catalysts, wherein other transition-metal ions are cosubstituted with Si-containing microporous architectures, is shown in Table 4 for a diverse range of acid-catalysed transformations.

The incorporation of a second metal can also tailor acidity in mesoporous frameworks and can help stabilise the primary metal into the framework (see Figure 10 and Table 4). The introduction of a divalent cation into a silicate framework will invoke a greater negative charge than a trivalent dopant; hence it will have to be compensated by a greater number of protons, therefore creating a stronger Brønsted acid site. In a related study involving bimetallic substitutions in mesoporous frameworks, the authors ${ }^{89}$ found that the divalent $\mathrm{Mg}$ substituted MCM-41 had lower acidity than its corresponding trivalent aluminium substituted analogue, as determined by temperature-programmed desorption of pyridine. This was attributed to the formation of $\mathrm{MgO}$ clusters during the calcination process, which blocked the acid sites. In contrast, the acidity of the bimetallic MgAl-MCM-41 was not hindered by this phenomenon, suggesting that the presence of $\mathrm{Al}$ in the framework stabilises isolated $\mathrm{Mg}$ (II) ions, creating a more efficient catalyst for the alkylation of $\mathrm{m}$-cresol due to its increased acidity.

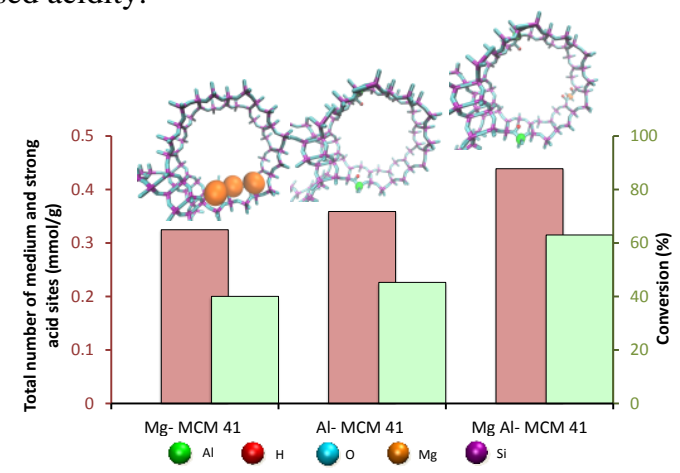

Figure 10: Tuning acidity in mesoporous bimetallic catalysts. ${ }^{82}$

While improvements can be achieved through bimetallic substitution it should be stressed that this is not a universal rule for all systems, and is in fact, the result of a prodigious design strategy that should take into consideration the nature and type of active sites in question as well as the surrounding pore architecture (for further examples see Table 4). We have already shown ${ }^{75}$ two examples of decreased catalytic outcomes by the incorporation of a second heteroatom (MgZnAlPO-5 in isopropylation of benzene and $\mathrm{ZnSiAlPO}-5$ in Beckmann rearrangement) and other related examples also exist in the literature $^{86}$ with analogous systems. One system which epitomises this point is MgSiAlPO-34, which was evaluated for the conversion of chloromethane to light olefins; an alternative to the industrially implemented MTO process. In this endeavour $^{86}$ a series of SiAlPO-34 catalysts were studied; in 
which varying amounts of magnesium were introduced into the SiAlPO-34 synthesis procedure, though the silicon quantities were kept constant. It was shown that by incorporating modest amounts of magnesium into the framework, the lifetime and activity of the catalyst could be markedly improved. However, the introduction of larger quantities of magnesium had the adverse effect of lowering the total acidity of the system, whilst promoting silicon islanding and limiting the catalytic performance. It was also observed that the quantity of silicon present in the catalyst was inversely proportional to the amount of magnesium incorporated. This observation suggests that the magnesium may have forced the silicon out of the framework, thereby leading to a less active species. This study highlights the delicate balance that exists in bimetallic catalysts, while simultaneously it emphasizes the need for a meticulous and prudent design approach coupled with the prerequisite for a detailed characterisation of the nature of the active site at the atomic level.

\section{Enhancing Synergy in Oxidation Reactions}

The profound link between the precise coordination geometry of a metal centre and its redox potential plays a crucial role in determining its reactivity, and subsequent catalytic potential. As such the aim to manipulate the exact nature of the active site together with its surrounding framework has prompted the design of a range of bimetallic AlPO catalysts for use in selective oxidation reactions. We have previously discussed (Figure 5) one example of this strategy employing the bimetallic Co(III)Ti(IV)AlPO-5 catalyst for the epoxidation of cyclohexene. ${ }^{4}$ By building on this concept, we have extended our study to include other redox-active metals; namely MnTiAlPO-5 and VTiAlPO-5. The latter catalyst showed a synergistic enhancement in catalytic potential for the epoxidation of cyclohexene, compared to its individual monometallic counterparts, under analogous conditions to the CoTiAlPO-5 (Figure 11A). The underlying nature of this catalytic synergy was explored further using a probe-based, in situ FT-IR study; in which the active acetyl peroxyborate (APB) oxidant was used as the molecular probe. This in situ study revealed that the combination of the Ti(IV) and vanadyl ions, undergoing simultaneous isomorphous substitution into the same AlPO-5 framework, aided the formation of the active peroxo species in the vicinity of the Ti(IV) centres, and improved the substrate-oxidant interactions. ${ }^{3}$ This resulted in a two-fold improvement in reactivity: as not only a more reactive active centre(s) is generated at the molecular level, but the concomitant advantageous interactions of the oxidant and activated substrate within the pores minimised wasteful oxidant decomposition, thereby facilitating a greater atom economy, higher product selectivity and better oxidant efficiency. ${ }^{3}$

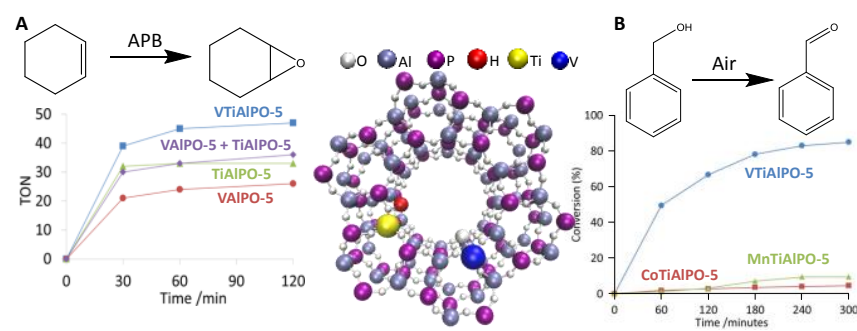

Figure 11: Modulating the potential of bimetallic active centres in selective oxidation reactions. ${ }^{3}$
In order to facilitate meaningful structure-property correlations and to understand the catalytic synergy at a more fundamental level, the exact nature of the active sites was probed through a combination of UV/Vis spectroscopy and EPR-HYSCORE (Electron Paramagnetic Resonance - Hyperfine Sub-level Correlation) techniques. It was found that ${ }^{3,74,90}$ on reduction of the catalyst some of the titanium species were able to access the Ti(III) state and had interestingly undergone framework substitution via a type I substitution mechanism (see Scheme 2 ). The vanadium species was similarly found to exclusively undergo type I substitution; despite vanadium occupying the $\mathrm{V}(\mathrm{V})$ oxidation state. The HYSCORE studies (Figure 7D) further revealed that the vanadium ions were found to exist as a vanadyl species $(\mathrm{V}=\mathrm{O})$, possessing an overall charge of just $3+$, hence its suitability for type I substitution. The EPR investigations were not able to conclusively substantiate the presence of $\mathrm{Ti}(\mathrm{IV})-\mathrm{O}-\mathrm{V}$ species, given the low levels of doping in the system, and further work is currently in progress to elucidate this. The effect of the various redox metals ( $\mathrm{V}-$, Tiand $\mathrm{Co}$ ) was catalytically probed by comparing the performance of the bimetallic VTiAlPO-5 catalyst with CoTiAlPO-5 and MnTiAlPO-5 in the aerobic oxidation of benzyl alcohol (Figure 11B). It was found that the performance of the VTiAlPO-5 catalyst far exceeded that of the other two systems. By simultaneously employing an in situ UV/Vis study, it was found that, unlike the VTiAlPO-5 catalyst, the MnTiAlPO-5 possessed a much higher fraction of titanium sites in an octahedral coordination, which could well be responsible for its inferior catalytic performance. It is therefore highly likely that the titanium sites in MnTiAlPO-5 would be coordinatively saturated and unable to interact efficiently with the oxidant and effectively generate the active oxidant species. Further, by contrasting the UV/Vis spectra of the oxidised and reduced samples it was found that the CoTiAlPO-5 catalyst had a significantly lower redox fraction than the VTiAlPO-5 system and this could have also played a significant role in hindering the activation of the oxidant, thereby limiting the reactivity with the CoTiAlPO-5 analogue (Figure 11B). The individual catalytic profiles (TON) for the monometallic and bimetallic analogues and the corresponding physical mixtures of the $\mathrm{V}$ and Ti-catalysts was also investigated for a different oxidation reaction - the epoxidation of olefins - and the trends from here were in sync with our earlier observations for the CoTiAlPO-5 catalysts (c.f. Figures 5B \& 11A). A selection of catalytic examples, highlighting analogous bimetallic synergy in microporous architectures and zeolites is shown in Table 5. It can be seen that the introduction of the multimetallic sites has proved effective in enhancing the catalytic performance in these materials.

Specific metal combinations can also promote synergistic interactions regardless of support architecture. Mesoporous MCM-41 molecular sieves have also been suitably modified with $\mathrm{V}$ and Ti metal ions. ${ }^{91}$ It was found that the inclusion of the titanium ion, in addition to the vanadium ion, resulted in a catalytic enhancement for styrene epoxidation, whilst the oxidation of toluene was markedly diminished (Figure 12). ${ }^{91}$ This clearly demonstrates that the addition of a second metal can have a dramatic influence on the properties of a monometallic catalyst and the strategy can be successfully extended to mesoporous architectures. A similar observation was found with a series of bimetallic Co-containing MCM-41 catalysts with different combinations of dopants either favouring the styrene epoxidation or the toluene oxidation (see 
Table 5: Emerging prospects for designed bimetallic solids in oxidation catalysis.

$\mathrm{C}=$ Conversion, $\mathrm{S}=$ Selectivity

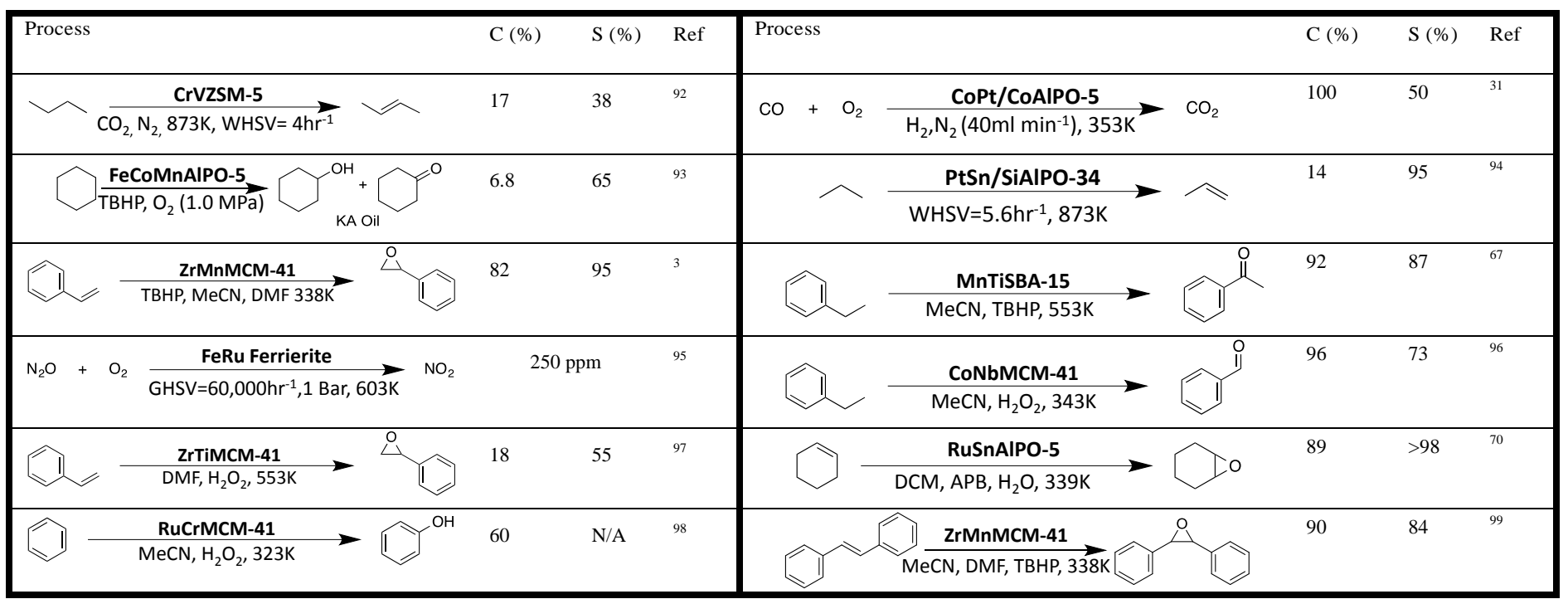

Table 5 for other examples). ${ }^{96}$ It is believed that the mode of activation is different for these individual reactions; side-chain functionalisation (epoxidation) is predominantly favoured with styrene, whilst activation of the $\mathrm{C}-\mathrm{H}$ bond (hydroxylation) is the main reaction with benzene.

Therefore, a detailed understanding of the mechanistic pathway in combination with meticulous design at the atomic level is paramount when choosing metal combinations to create specific active sites. This study further emphasises the need to rationally combine metal substituents using all of facets that have been discussed above (ionic size, coordination geometry, redox potential, type of substitution, etc.), so that the design and combination approach at the molecular level will result in distinctive and subtle modifications to active site, often translating into startling differences in catalytic activity and selectivity.

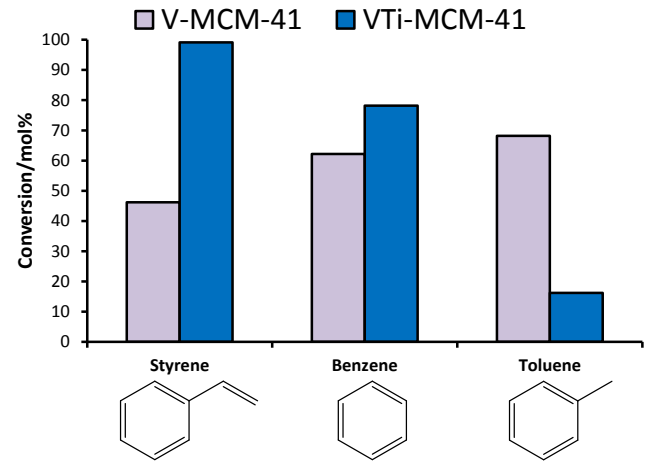

Figure 12: Contrasting the effects of bimetallic substitution in mesoporous solids - substrate versatility. ${ }^{91}$

\section{Other reactions demonstrating catalytic synergy}

The benefits of generating multimetallic (and multifunctional) catalysts can also be realised in reduction reactions. Simultaneous $\mathrm{NO}_{\mathrm{x}}$ reduction and VOC oxidation is an attractive concept, as it offers the potential to significantly reduce costs for pollutant abatement. $\mathrm{Cu}(\mathrm{II})$ is very versatile, as it possess both redox and acidic properties, and has subsequently been found to be highly active in $\mathrm{NO}_{\mathrm{x}}$ reduction. ${ }^{100}$ However, $\mathrm{Cu}$ (II) species (preferentially occupying square planar or octahedral geometries) were found to be unstable in the MCM-41 framework forming inactive $\mathrm{CuO}$ species on calcination, as characterised by EPR and UV-Vis DRS studies. ${ }^{100}$ From a design perspective, this was overcome by introducing a small quantity aluminium ions into the framework, which was found to stabilise a large proportion of the $\mathrm{Cu}$ (II) ions (Figure 13). Consequently the bimetallic catalyst showed a higher activity and increased stability, in comparison to the monometallic analogue. In their synthetic approach, the authors have taken specific precautions to insert conservative amounts of $\mathrm{Cu}$ (II) into the framework. It was found that ${ }^{100}$ while large quantities of $\mathrm{Cu}$ (II) can lead to a higher density of isolated, active $\mathrm{Cu}$ (II) sites, it also reduces the hydrophobic character and hence, in the presence of water, the conversion is significantly compromised.

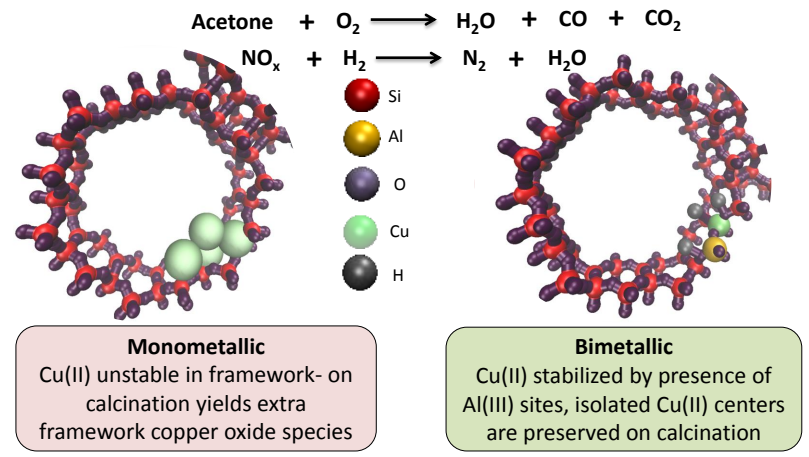

Figure 13: Contrasting the nature and geometry of the $\mathrm{Cu}(\mathrm{II})$ site in mono- and bimetallic mesoporous solids. ${ }^{100}$

\section{Computational design of active sites}

While the desire to develop and enhance catalyst design through bimetallic substitution is being driven forward experimentally, the notion is also being developed through parallel theoretical studies. ${ }^{76,80}$ Recently Cora et al have 
published a comprehensive study on the mechanism by which the monometallic MnAlPO-5 catalyst is able to aerobically activate ethane to produce a range of oxygenated products. This was built on previous studies ${ }^{35,36}$ demonstrating the regioselective oxidation of alkanes. The theoretical study of Cora et al concluded that the pre-activation step (breaking of the first $\mathrm{C}-\mathrm{H}$ bond) was the most energetically demanding step in the whole pathway. By repeating the initial stage of this study (pre-activation) with FeAlPO-5 instead of MnAlPO-5, subtle differences in the reaction pathway and energetics were observed. ${ }^{101}$ It was shown that as manganese displays a greater preference for the $\mathrm{M}$ (II) state, it was less energetically demanding for $\mathrm{Mn}$ (III) to undergo reduction to $\mathrm{Mn}$ (II), whilst simultaneously activating the alkane via an oxidative transfer. It was also notable that manganese was able to activate the hydrocarbon with greater ease than the iron-analogues. Furthermore, once reactive intermediates were formed, an alternative pre-activation mechanism was accessible (secondary pre-activation). Manganese was also found to accomplish this step at a significantly lower energy cost. It is only when considering the propagation step that the benefits of iron become apparent (Figure 14). In this step, the metal centre is being oxidized from $\mathrm{M}$ (II) to $\mathrm{M}$ (III), whilst producing oxygenated hydrocarbon products; and the iron sites are able to perform this step with much greater ease than manganese. From this work, ${ }^{101}$ it was subsequently argued that the complimentary behavior of these two different oxidation sites would make them appropriate candidates to unite to form a bimetallic FeMnAlPO-5 catalyst, should one wish to optimize this reaction further. Such theoretical advances significantly influence the molecular design of bimetallic and multifunctional active sites at the atomic level, which facilitate meaningful structure-property relationships to be established in our quest for effecting stimulating catalytic challenges.

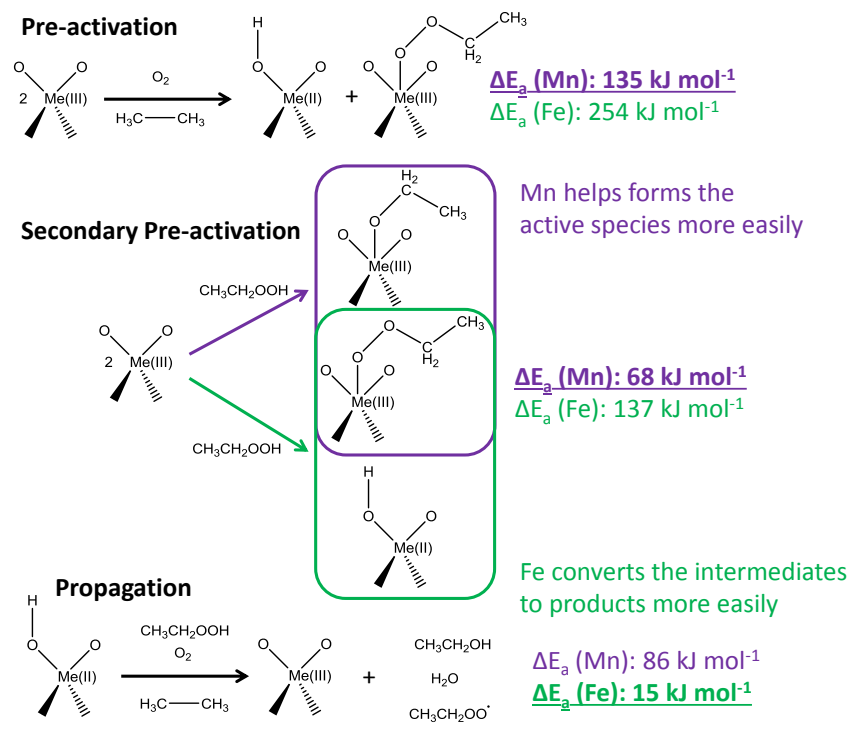

Figure 14: Complementary behaviour of $\mathrm{Mn}$ and $\mathrm{Fe}$ active centres in AlPO-5 catalysts for alkane oxidation. ${ }^{101}$

\section{Future Prospects: Designed Single-Sites in Hybrid Hierarchical Architectures}

Despite the vast range of subtle manipulation techniques employed in the synthesis of microporous materials, which specifically aim to alter and optimise the catalytic activity, some fundamental shortcomings still exist. In some cases, especially with bulky substrates or intermediate transitionstates, the presence of the micropores can result in poor masstransfer, short life times, hindered diffusion and limited substrate scope. Mesoporous molecular sieves have been designed to circumvent these issues; but due to their amorphous framework they are often less stable, not amenable to isomorphous substitution to the same degree as their microporous counterparts, possess less well-defined redox and acidic active centres and are therefore less compliant in their industrial application. As a compromise between the two, hierarchical molecular sieves can offer a good degree of scope to address some of the above challenges. The concept of designed microporous architectures possessing well-defined active sites that can be manipulated at the atomic level, coupled with the added advantage of an auxiliary mesoporous network to aid mass-transfer, holds great potential in the engineering of novel single-site heterogeneous catalysts. By employing a dual templating technique (combining design principles from microporous and mesoporous molecular sieves), one can preferentially bestow highly active microporous single-sites with a reduced induction period and longer lifetime, owing to the presence of the associated mesopores within the same catalyst. ${ }^{102}$ As such hierarchical molecular sieves have much to offer, combining the high selectivity, versatility and stability that can be associated with isomorphous metal substitution at the nanoscale (atom-specificity), coupled with their propensity for large pore volumes and high surface areas for the improved diffusion of molecules through the framework. Early research has already highlighted ${ }^{102,103}$ the ability of the hierarchical systems to circumvent the diffusion limitations imposed by the conventional microporous frameworks, which also includes the incorporation of a mesoporous network into monometallic AlPOs.

Over the last few years a range of synthetic approaches for the creation of secondary porosity within framework architectures have been developed with their own specific advantages (Figure 15).
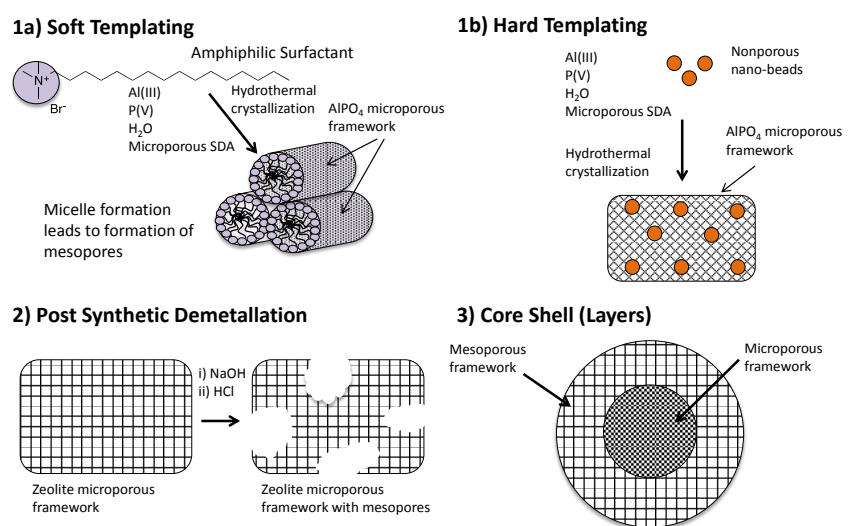

Figure 15: Outline of strategies for design and synthesis of hierarchical molecular sieves.

In order to selectively catalyse a reaction utilising a porous catalyst it is paramount that the active sites that are located within the internal pores and channels are accessible to the substrate. When trying to activate bulky substrate molecules this can be a concern, particularly in microporous systems. To improve internal diffusion of such species, hierarchically 
porous SiAlPO-5 (HP-SiAlPO-5) catalysts were specifically synthesised for the alkylation of benzene with benzyl alcohol. ${ }^{104}$ Despite TPD-TGA of n-propylamine indicating that both the microporous and hierarchical catalysts had near identical acidity characteristics, the hierarchical catalyst was three times more active than the microporous analogue. The marked difference in the catalytic performance suggests that the presence of the mesopores had a beneficial effect in improving the rate of the reaction. However, it is important to note that the presence of an undesired dense phase in the hierarchical sample was detected by XRD. ${ }^{29} \mathrm{Si}$ MAS NMR and EDX further confirmed the presence of an additional amorphous phase; highlighting the challenges associated with the synthesis of phase-pure hierarchical materials. With this in mind, the activity of both systems was compared to an amorphous silicaalumina (ASA) phase material. It was interesting to note that the ASA phase was not able to emulate the activity of the HPSiAlPO-5 catalyst, but it did provide a catalytic profile that was superior to the microporous SiAlPO-5 catalyst. These findings, however, emphasised the importance of creating mesopores for enhancing the catalytic ability of bulky substrates, as they facilitate greater access to the active site. However, further work is required from a synthetic standpoint, in order to understand the nature of the acid sites at a molecular level, for which phase-pure materials with well-ordered mesopores are a pre-requisite.

Microporous AlPOs containing isomorphously-substituted redox metals have been shown to be effective catalysts in oxidation reactions; though they can suffer from limited substrate scope owing to restrictions in access to the active sites. Hierarchical systems have been shown to facilitate the access of bulky molecules to the active site and hence, increase the substrate scope to include building blocks for pharmaceuticals and fine-chemicals. Monometallic hierarchical $\mathrm{Mn}$ and Fe molecular sieves (HP-MnAlPO-5 and HP-FeAlPO5) were synthesised with a pore-size distribution between 5-30 nm. ${ }^{105}$ The oxidation of hydrocarbons was utilised ${ }^{105}$ to test the efficacy of the hierarchical catalysts compared to their microporous analogues. It was found ${ }^{105}$ that the increased activity of the hierarchical system was dependent on substrate size (Figure 16A). The introduction of the mesopores into the microporous MnAlPO-5 catalyst improved its catalytic activity dramatically for the oxidation of indan, moderately for cyclohexene and only slightly for fluorene. In the case of cyclohexene and indan, the molecules are sufficiently small to permit diffusion into the micropores of AlPO-5. Subsequently they can access the internal active sites and be subjected to various oxidation pathways to yield functionalised products. The presence of the auxiliary mesopore network can lead to an increase in the mass-transfer of the reactants to the active sites, simultaneously enhancing the diffusion of reactants and egress of products, thereby facilitating an increase in the rate of conversion. This is particularly dramatic in the case of indan, as its molecular dimensions are very similar $(4.8 \times 6.4 \AA)$ to that of the micropore size, whilst cyclohexene $(4.8 \times 4.7 \AA)$ is much smaller than the micropore aperture $(7.3 \AA)$. In contrast, fluorene $(5.1 \times 8.8 \AA)$ cannot fit into the micropores and subsequently the reaction can only occur on external active sites. In this case, internal diffusion is not the limiting factor, hence the conversion of the hierarchical and microporous systems are comparable. The combination of micropores and mesopores lead to a dramatic improvement in conversion when the substrate is of comparable size to the micropore, indicating that the active site is in the micropore and the mesopores are purely there to facilitate the role of diffusion.

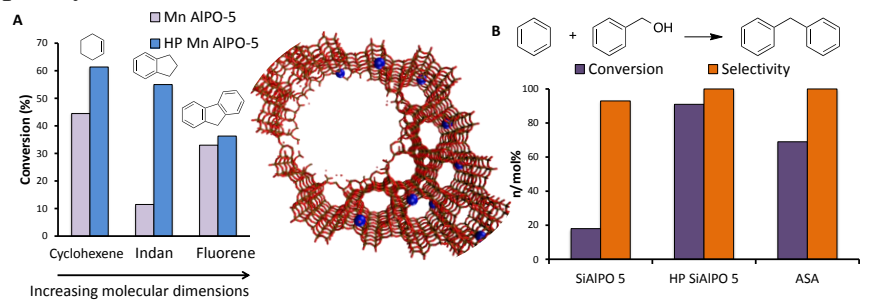

Figure 16: Redox (A) and acid catalysis (B) utilizing hierarchical MAlPO molecular sieves. ${ }^{105,107}$

Conventionally, purely mesoporous materials can exhibit lowstability due to the mesopore walls being comprised of crosslinked amorphous silica. Generating mesopores alongside a microporous scaffold can also be advantageous for stabilising certain types of active species that are otherwise not stable in purely microporous or mesoporous environments. $\mathrm{Cr}$ containing molecular sieves have been reported to be an efficient oxidation catalyst, but the low-stability and the propensity for $\mathrm{Cr}$ to leach from the porous network has prevented it wider use. ${ }^{106}$ It has been reported ${ }^{107}$ that a hierarchical porous CrAlPO-5 framework has been designed, which retained and stabilised the active chromium sites of CrMCM-41, but incorporated them into a stable hierarchical AlPO-5 framework. ${ }^{107}$ Powder-XRD confirmed that AlPO-5 was the sole microporous phase present, while BET confirmed the presence of mesopores. SEM micrographs showed uniform monophasic particles confirming only one phase (bimodal, hierarchical) existed. The efficacy of the hierarchically porous sample and microporous CrAlPO-5 catalyst was evaluated in the oxidation of the bulky substrate tetralin, to 1-tetralone. It was revealing that the yields were enhanced by the presence of the mesopores in the hierarchical system, compared to the microporous analogue. This indicated that the generation of stable, active chromium sites enhances the catalytic oxidation reaction, not only by improving accessibility of substrates and products through the availability of mesopores; but stabilisation of the active centre leads to sustained catalytic performance.

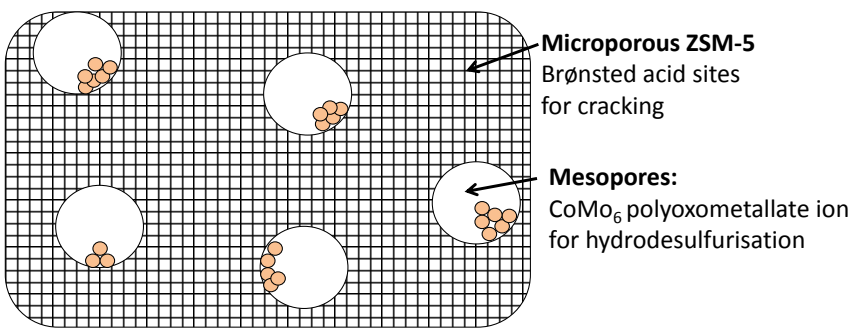

Figure 17: Bifunctional hierarchical ZSM-5 catalysts for combined hydrocracking and hydrodesulfurisation. ${ }^{108}$

As well as improving diffusion and mass-transfer to- and fromthe active site, hierarchical molecular sieves can also combine diverse microporous active sites with a wider range of catalytic function; e.g. creating bifunctional and multifunctional catalysts. An example of this simultaneously utilises the ability of mesopores to incorporate bulkier active sites (e.g. nanoclusters or metal complexes) within the framework, with the concomitant ability of microporous architecture to adroitly introduce well-defined acid sites. These two qualities can be elegantly exploited in hierarchically porous materials, by combining a Brønsted acid site in the micropores (as illustrated 
earlier) ${ }^{71,75}$ with adeptly-designed nanoparticles in the mesopores. This was demonstrated ${ }^{108}$ with hierarchically porous ZSM-5, in which $\mathrm{CoMo}_{6}$ polyoxometallate ions were housed within the mesopores, and the micropores provided Brønsted acidity (Figure 17). This bifunctional hierarchically porous zeolite was utilised for the simultaneous hydrocracking and hydrodesulfurisation process. This system is particularly advantageous as the hydrodesulfurisation reaction was able to occur in close proximity to the cracking (creating the desired transition-state), resulting in a greater hydrodesulfurisation activity. This exciting concept ${ }^{108}$ can now be potentially extended to other systems to facilitate one-pot cascade reactions with high activities and selectivities. These initial studies and many others ${ }^{103-108}$ that underpin the potential application of hierarchical molecular sieves in catalysis, have been promising and have highlighted the ability of the hierarchical systems to circumvent the mass-transfer problems that have plagued catalytically promising microporous molecular sieves. Recent advances $3,67,71,75,97$ in creating well-defined, multifunctional single-sites at the atomic level on microporous architectures need to be suitably integrated and adapted for the design and synthesis of hybrid hierarchical systems. This needs to be harmonised with current developments, incorporating in situ spectroscopic and operando techniques, for a better quantitative understanding of the nature of the active sites at the molecular level, for the successful implementation and adaptation of these catalysts.

\section{Conclusion}

In this Feature Article we have demonstrated the benefits and future potential associated with creating discrete, well-defined multifunctional active sites in porous architectures. By aligning the synthetic strategy with in situ spectroscopic and operando methods for probing the nature of the active site at the molecular level, we have demonstrated how these isolated single-sites can communicate with one another, at the atomic scale, to provide synergistic enhancements in catalytic activity and selectivity. These characterization tools have afforded robust structure-property correlations to be established, which have enabled the rational design of highly active and selective catalysts for selective oxidation and acid-catalysed processes. We have set the stage for the integration and amalgamation of these developments leading to the discovery and design of novel hierarchical materials that can help overcome masstransfer limitations and lead to the stabilisation of otherwise unstable active centres in porous architectures. It is envisaged that by combining the advantages of the design-application approach, coupled with a fundamental understanding of the mechanistic characteristics at the atomic scale, can lead to the design and development of the next generation of multifunctional, single-site heterogeneous catalysts.

\section{Acknowledgements}

We would like to thank our colleagues in Italy, Drs E. Gianotti, M. Manzoli and M. Chiesa for their stellar contributions and stimulating scientific discussions to the original research. Our industrial collaborators and partners, Drs A. Levy, S. Keenan, S. Bare and T. Mezza from Honeywell, are acknowledged for their intellectual input and assistance with the operando spectroscopy tools. Finally, we are grateful to Honeywell LLC for their continued financial support that has facilitated this research.

\section{Notes and references}

a School of Chemistry, University of Southampton, Highfield, Southampton, SO17 1BJ UK. R.Raja@ soton.ac.uk

1 J. M. Thomas and R. Raja, Chem. Commun., 2001, 675-687.

2 J. M. Thomas, R. Raja and D. W. Lewis, Angew. Chem. Int. Ed., 2005, 44, 6456-6482.

3 R. M. Leithall, V. N. Shetti, S. Maurelli, M. Chiesa, E. Gianotti and R. Raja, J. Am. Chem. Soc., 2013, 135, 2915-2918.

4 J. Paterson, M. Potter, E. Gianotti and R. Raja, Chem. Commun., 2011, 47, 517-519.

$5 \quad$ European Chemical News, 1995, 18, 20.

6 A. Chauvel, B. Delmon and W. F. Höelderich, Appl. Catal. A, 1994, 115, 173-217.

7 W. F. Höelderich, Stud. Surf. Sci. Catal., 1989, 46, 193-209.

8 K. Tanabe and T. Nishizaki, Proc. VIth Int. Congr. Catal., 1977.

9 W. F. Höelderich, Proceedings of the 10th International Congress on Catalysis, 1993.

10 J. N. Armor, Appl. Catal., 1991, 78, 141-173.

11 European Chemical News, 1994, 12, 26.

12 R. A. Asuquo, G. Eder-Mirth and J. Lercher, J. Catal., 1995, 115, $376-$ 382 .

13 J. A. Cusumano, Chemtech, 1992, 22, 482-489.

14 Y. Izumi, H. Ichihashi, Y. Shimazu, M. Kitamura and H. Sato, Bull. Chem. Soc. Jpn, 2007, 80, 1280-1287.

15 B. Guo, L. Zhu, X. Hu, Q. Zhang, D. Tong, G. Li and C. Hu, Catal. Sci. Technol., 2011, 1, 1060-1067.

16 M. G. Clerici, G. Bellussi and U. Romano, J. Catal., 1991, 129, 159167.

17 G. C. Chinchen, K. Mansfield and M. S. Spencer, Chem. Technol., 1990, 20, 692-699.

18 B. L. Goodall, Transition Metal Catalyzed Polymerizations, Harwood Academic Publishers, 1983.

19 K-H. Daum, J. Storch and J. Rieder, US Pat., 7,704,476, 2010.

20 B. C. Gates and H. Topsoe, Polyhedron, 1997, 16, 3213-3217.

21 M. T. Mchea III and R. M. Yarrington, US Pat., 4,863,707, 1989.

22 K. Kochloef, Handbook of Heterogeneous Catalysis, Wiley/VCH, Weinheim, 1997.

23 F. Sperner and W. Hohmann, Platinum Metals Rev, 1976, 20.

24 M. Roussel, M. Bouchard, E. Bordes-Richard, K. Karim and S. AlSavari, Catal. Today, 2005, 99, 77-87.

25 R. P. Nielsen, J. H. La Rochelle, US Pat., 3,962,136, 1976.

26 K.D. Loos, K. C. McCullough, D. Q. Whitworth, US Pat., 4,128,622, 1978 .

27 S. T. Wilson, B. M. Lok, C. A. Messina, T. R. Cannan and E. M. Flanigen, J. Am. Chem. Soc., 1982, 104, 1146-1147.

28 P. T. Barger, US Pat., 5,095,163, 1992.

29 M. Hartmann and L. Kevan, Chem. Rev., 1999, 99, 635-663.

30 M. Anilkumar and W. Hoelderich, Catal. Today, 2012, 198, 289-299.

31 C. X. Wang, L. Zhang and Y. Liu, Appl. Catal. B:Envi., 2013, 136137, 48-55.

32 Z. Nawaz and W. Fei, Ind. Eng. Chem. Res., 2009, 48, 7442-7447.

33 T. Komatsu and H. Ikenaga, J. Catal., 2006, 241, 426-434.

34 M. D. Jones, R. Raja, J. M. Thomas, B. F. G. Johnson, D. W. Lewis, J. Rouzand and K. D. M. Harris, Angew. Chem. Int. Ed., 2003, 42, 43264331.

35 J. M. Thomas, R. Raja, G. Sankar and R. G. Bell, Nature, 1999, 398, 227-230.

36 R. Raja, G. Sankar and J. M. Thomas, Angew. Chem. Int. Ed., 2000, 39, 2313-2316.

37 A. Corma, J. Díaz-Cabañas, J. Martinez-Triguero, F. Rey and F. Rius, Nature, 2002, 418, 514-517.

38 S. T. Wilson, B. M. Lok and E. M. Flanigen, US Pat., 4,310,440, 1982.

39 M. E. Davis, C. Saldarriaga and C. Montes, Zeolites, 1988, 8, 362-366.

40 M. Estermann, L. B. McCusker, C. Baerlocher, A. Merrouche and H. Kessler, Nature, 1991, 352, 320-323.

41 M. E. Davis, Nature, 2002, 417, 813-821. 
42 J. S. Beck, J. C. Vartuli, W. J. Roth, M. E. Leonowicz, C. T. Kresge, K. D. Schmitt, C. T. W. Chu, D. H. Olson, E. W. Sheppard, S. B. McCullen, J. B. Higgins and J. L. Schlenker, J. Am. Chem. Soc., 1992, 114, 10834-10843.

43 D. Zhao, J. Feng, Q. Huo, N. Melosh, G. H. Fredrickson, B. F. Chmelka and G. D. Stucky, Science, 1998, 279, 548-552.

44 S. Inagaki, A. Koiwai, N. Suzuki, Y. Fukushima and K. Kuroda, Bull. Chem. Soc. Jpn., 1996, 69, 1449-1457.

45 F. Farges and G. E. Brown Jr, Geochim. Cosmochim. Acta, 1997, 61, $1863-1870$.

46 P. E. Sinclair, G. Sankar, C. R. A. Catlow, J. M. Thomas and T. Maschmeyer, J. Phys. Chem. B, 1997, 101, 4232-4237.

47 T. Blasco, A. Corma, M. T. Navarro and J. Pérez-Pariente, J. Catal., 1995, 156, 65-74.

48 M. A. Roberts, G. Sankar, J. M. Thomas, R. H. Jones, H. Du, J. Chen, W. Pang and R. Xu, Nature, 1996, 381, 401-404.

49 M. W. Anderson, O. Terasaki, T. Ohsuna, A. Phillippou, S. P. Mackay, A. Ferreira, J. Rocha and S. Lidin, Nature, 1994, 367, 347351.

50 A. B. Hungria, R. Raja, R. D. Adams, B. Captain, J. M. Thomas, P. A. Midgley, V. Golovko and B. F. G. Johnson, Angew. Chem. Int. Ed., 2006, 4782-4785.

51 R. D. Adams, E. M. Boswell, B. Captain, A. B. Hungria, P. A. Midgley, R. Raja and J. M. Thomas, Angew. Chem. Int. Ed., 2007, 8182-8185.

52 R. Raja, R .D. Adams, D. A. Blom, W. C. Pearl Jr., E. Gianotti and J. M. Thomas, Langmuir, 2009, 25, 7200-7204.

53 M. D. Hughes, Y.-J. Xu, P. Jenkins, P. McMorn, P. Landon, D. I. Enache, A. F. Carley, G. A. Attard, G. J. Hutchings, F. King, E. H. Stitt, P. Johnston, K. Griffin and C. J. Kiely, Nature, 2005, 1132-1135.

54 R. D. Adams, M. Chen, G. Elpitiya, M. E. Potter and R. Raja, ACS. Catal., 2013, 3, 3106-3110.

55 C. S. Hinde, S. V. Aswegen, G. Collins, J. D. Holmes, T. S. A. Hor and R. Raja, Dalton Trans., 2013, 42, 12600-12605.

56 P. Roffia, M. Padovan, F. Leofanti, M. A. Mantegazza, G. De Alberti and G. R. Tamazik, US Pat., 4,794,198, 1988.

57 I. Q. Chen, A. Bozzano, B. Glover, T. Euglerud and S. Kvisle, Catal. Today, 2005, 106, 103-107.

58 M. Moliner, Y. Román-Leshkov and M. E. Davis, Proc. Natl. Acad. Sci., 2010, 107, 6164-6168.

59 M. Renz, T. Blasco, A. Corma, V. Fornés, R. Jensen and L. Nemeth, Chem. Eur. J., 2002, 8, 4708-4717.

60 A. Corma, P. Esteve and A. Martinez, J. Catal., 1996, 161, 11-19.

61 R. Raja and A. J. Paterson, US Pat., US20130245322, 2013.

62 R.Raja and J. M. Thomas, US Pat., US20130245323, 2013.

63 R. Raja, G. Sankar and J. M. Thomas, J. Am. Chem. Soc., 2001, 123, 8153-8154.

64 J. M. Thomas and R. Raja, Proc. Natl. Acad. Sci., 2005, 102, 1373213736 .

65 A. B. Levy, R. Raja and M. E. Potter, US Pat., US20130109851, 2013.

66 A. J. Paterson, PhD Thesis, University of Southampton, 2011.

67 P. Visuvamithiran, K. Shanthi, M. Palanichamy and V. Murugesan, Catal. Sci. Technol., 2013, 3, 2340-2348.

68 J. M. Thomas, C. R. A. Catlow and G. Sankar, Chem. Commun., 2002, 24, 2921-2925.

69 M. Selvaraj, S. W. Song and S. Kawi, Micro. Meso. Mater., 2008, 110 , 472-479.

70 M. E. Potter, A. J. Paterson and R. Raja, ACS Catal., 2012, 2, 24462451

71 E. Gianotti, M. Manzoli, M. E. Potter, V. N. Shetti, D. Sun, A. J. Paterson, T. M. Mezza, A. Levy and R. Raja, Chem. Sci., 2014, in press.

72 K. Simmance, G. Sankar, R. G. Bell, C. Prestipino and W. van Beek, Phys. Chem. Chem. Phys., 2010, 12, 559-562.

73 A. Brückner, Chem. Soc. Rev., 2010, 39, 4673-4684.

74 S. Maurelli, M. Vishnuvarthan, M. Chiesa, G. Berlier and S. Van Doorslaer, J. Am. Chem. Soc., 2011, 133, 7340-7343.

75 M. E. Potter, D. Sun, E. Gianotti, M. Manzoli and R. Raja, Phys. Chem. Chem. Phys., 2013, 15, 13288-13295.

76 L. Gomez-Hortiguela, F. Cora, G. Sankar, C. M. Zicovich-Wilson and C. R. A. Catlow, Chem. Eur. J., 2010, 16, 13638-13645

77 S. P. Bates and R. A. Van Santen, Adv. Catal., 1998, 42, 1-114.
78 A. Vinu, M. Karthik, M. Miyahara, V. Murugesan and K. Ariga, $J$. Mol. Catal. A: Chem., 2005, 230, 151-157.

79 M. Karthik, A. K. Tripathi, N. M. Gupta, A. Vinu, M. Hartmann, M Palanichamy and V. Murugesan, Appl. Catal. A: Gen., 2004, 268, 139149.

80 F. Cora, M. Alfredsson, M. Barker, R. G. Bell, M. D. Foster, I. Saadoune, A. Simperler and C. R. A. Catlow, J. Solid State Chem., 2003, 176, 496-529.

81 P. Lian, W. Geo, F. Yan, W. Liu, G. Li, H. Wang and G. Sun, Catal. Today, 2002, 74, 137-143.

82 A. Vinu, K. Usha Nandhini, V. Murugesan, W. Böhlmann, V. Umamaheswari, A. Pöppl and M. Hartmann, Appl. Catal. A: Gen., 2004, 265, 1-10.

83 M. Lefenfeld, R. Raja, A. J. Paterson and M. E. Potter, US Pat., US20100249476, 2010.

84 D. R. Dubois, D. L. Obrzut, J. Liu, J. Thundimadathil, P. M. Adekkanttu, A. Punnoose and M. S. Seehra, Fuel Proc. Tech., 2003 , 83, 203-218.

85 I. Eswaramoorthi and N. Lingappan, Appl. Catal. A:Gen., 2003, 245, 119-135.

86 D. Zhang, Y. Wei, L. Xu, F. Chang, Z. Liu, S. Meng, B. L. Su and Z. Liu, Micro. Meso. Mater., 2008, 116, 684-692.

87 A. Geetha Bhavani and A. Pandurangan, J. Mol. Catal. A:Chem., 2007, 267, 209-217.

88 G. Sastre, D. W. Lewis and C. R. A. Catlow, J. Phys. Chem. B., 1997, 101, 5249-5262.

89 A. Vinu, K. Ariga, S. Saravanamurugan, M. Hartmann and V. Murugesan, Micro. Meso. Mater., 2004, 76, 91-98.

90 S. Maurelli, M. Chiesa, E. Gianotti, R. M. Leithall and R. Raja, Chem. Commun., 2012, 48, 8700-8702.

91 V. Parvulescu, C. Anastasescu, C. Constantin and B. L. Su, Catal. Today, 2003, 78, 477-485.

92 B. P. Ajayi, R. Jermy, B. A. Abussaud and S. Al-Khattaf, J. Porous Mater., 2013, 20, 1257-1270.

93 L. Zhou, J. U. H. Miao, X. Li and F. Wang, Catal. Lett., 2005, 99, 231-234.

94 Z. Nawaz, X. Tang, Q. Zhang, D. Wang and W. Fei, Catal. Commun., 2009, 10, 1925-1930.

95 G. D. Pirngruber, L. Frunz and J. A. Z. Pieterse, J. Catal., 2006, 243, 340-349.

96 V. Pârvulescu, C. Tablet, C. Anastasescu and B. L. Su, Catal. Today 2004, 93-95, 307-313.

97 Y. Wang, Y. Guo, G. Wang, Y. Liu and F. Wang, J. Sol-Gel Sci. Technol., 2011, 57, 185-192.

98 V. Pârvulescu, C. Anastasescu and B. L. Su, J. Mol. Catal. A: Chem., 2004, 211, 143-148.

99 M. Selvaraj, K. S. Seshadri, A. Pandurangan and T. G. Lee, Micro. Meso. Mater., 2005, 79, 261-268.

100 M. Karthik, L.-Y. Lin and H. Bai, Micro. Meso. Mater., 2009, 117, 153-160.

101 L. Gomez-Hortiguela, F. Cora and C. R. A. Catlow, Phys. Chem Chem. Phys., 2013, 15, 6870-6874.

102 K. Na, M. Choi and R. Ryoo, Micro. Meso. Mater., 2013, 166, 3-19.

103 C. M. A. Parlett, K. Wilson and A. Lee, Chem. Soc. Rev., 2013, 42, 3876-3893.

104 N. Danilina, F. Krumeich and J. A. van Bokhoven, J. Catal., 2011, 168, 80-85.

105 L. Zhou, T. Lu, J. Xu, M. Chen, C. Zhang, C. Chen, X. Yang and J. Xu, Micro. Meso. Mater., 2012, 161, 76-83.

106 R. A. Sheldon, M. Wallau, I. W. C. E. Arends and U. Schuchardt, Acc. Chem. Res., 1998, 31, 485-493.

107 J. Kim, S. Bhattacharjee, K. Jeong, S. Jeong, M. Choi, R. Ryoo and W. Ahn, New. J. Chem., 2010, 34, 2971-2978.

108 K. T. Hojholt, P. N. R. Vennestrom, R. Tiruvalam and P. Beato, Chem. Commun., 2011, 47, 12864-12866. 